\title{
On Some Circular Distributions Induced by Inverse Stereographic Projection
}

\author{
Shamal Chandra Karmaker
}

\author{
A Thesis \\ for The Department of \\ Mathematics and Statistics
}
Presented in Partial Fulfillment of the Requirements for the Degree of Master of Science (Mathematics) at Concordia University Montréal, Québec, Canada

November 2016

(c) Shamal Chandra Karmaker 2016 


\section{CONCORDIA UNIVERSITY \\ School of Graduate Studies}

This is to certify that the thesis prepared

By: Shamal Chandra Karmaker

Entitled: On Some Circular Distributions Induced by Inverse Stereographic

Projection and submitted in partial fulfillment of the requirements for the degree of

\section{Master of Science (Mathematics)}

complies with the regulations of the University and meets the accepted standards with respect to originality and quality.

Signed by the final examining committee:

Examiner

Dr. Arusharka Sen

Examiner

Dr. Wei Sun

Thesis Supervisor

Dr. Y.P. Chaubey

Thesis Co-supervisor

Dr. L. Kakinami

Approved by

Chair of Department or Graduate Program Director

Dean of Faculty

Date 


\section{Abstract \\ On Some Circular Distributions Induced by Inverse Stereographic Projection}

\section{Shamal Chandra Karmaker}

In earlier studies of circular data, mostly circular distributions were considered and many biological data sets were assumed to be symmetric. However, presently interest has increased for skewed circular distributions as the assumption of symmetry may not be meaningful for some data. This thesis introduces three skewed circular models based on inverse stereographic projection, introduced by Minh and Farnum (2003), by considering three different versions of skewed- $t$ considered in the literature, namely Azzalini skewed- $t$, two-piece skewed- $t$ and Jones and Faddy skewed- $t$. Shape properties of the resulting distributions along with estimation of parameters using maximum likelihood are discussed in this thesis. Further, three real data sets (Bruderer and Jenni, 1990; Holzmann et al., 2006; Fisher, 1993) are used to illustrate the application of the new model and its extension to finite mixture modelng. Goodness of fit of the new distributions is studied using maximum log-likelihood, Akaike information criterion and chi-square values. It is found that Azzalini and JonesFaddy skewed- $t$ versions are good competitors; however the Jones-Faddy version is computationally more tractable. 


\section{Acknowledgements}

Foremost, I would like to express my deepest gratitude to my supervisor, Dr. Yogendra P. Chaubey. His kindness, guidance and patience throughout my graduate studies have been enormously helpful and without him I would not have made it through the program.

I would like to thank my Co-supervisor, Dr. Lisa Kakinami, for her kind support and guidance throughout the program.

I would also like to thank the members of the Statistics group at Concordia University for helpful discussion and advice. Thank you, as well, to all the wonderful professors I had at Concordia University, who helped me to further enhance my knowledge in Statistics. I thank Concordia University for accepting me and the financial support.

Last but not least, I would like to thank all my family members for their unconditional help and support, specially my wife Bipasha Singha for providing her continuous encouragement. This journey would have never be completed without anyone of you. 


\section{Contents}

List of Tables ...................... . . viii

List of Figures . . . . . . . . . . . . . . . . . . $\mathrm{x}$

1 Introduction 1

1.1 Introduction . . . . . . . . . . . . . . . . 1

1.2 Outline of the thesis . . . . . . . . . . . . . . 2

1.3 Circular data and sample statistics . . . . . . . . . . . . . 2

1.4 Parametric distributions on circle . . . . . . . . . . . . . 10

1.4.1 The uniform distribution . . . . . . . . . . . . . . . 11

1.4.2 The von Mises (VM) distribution . . . . . . . . . . . . . 11

1.4.3 Cardioid distribution . . . . . . . . . . . . . . 12

1.4.4 Wrapped distribution ............... . . 12

1.4.5 Skewed distributions . . . . . . . . . . . . 15

1.5 Literature review . . . . . . . . . . . . . . . . . . . . . . . . . . 18

2 Generation of Circular Distributions $\quad 21$

2.1 Introduction . . . . . . . . . . . . . . . . . . . . . . . 21

2.2 Methods of generating circular distributions . . . . . . . . . . . 21

3 Inverse Stereographic Projection Circular Skewed- $t$ Distributions 27

3.1 Introduction . . . . . . . . . . . . . . . . . . 27

3.2 Skewed- $t$ distribution on the real line . . . . . . . . . . . . . . . 28

3.2.1 The Jones and Faddy (2003) skewed-t distribution . . . . . . 28 
3.2.2 The Azzalini skewed- $t$ distribution _ . . . . . . . . . . . . 29

3.2.3 The two-piece skewed- $t$ distribution $\ldots \ldots \ldots \ldots$

3.3 Inverse Stereographic Projection (ISP) skewed-t distribution . . . . 30

3.3.1 Jones and Faddy ISP skewed- $t$ distribution . . . . . . . . . 30

3.3.2 Azzalini type ISP skewed- $t$ distribution . . . . . . . . . . 33

3.3.3 Two-piece ISP skewed-t distribution . . . . . . . . . . . 34

4 Shape Characteristics of ISP Skewed- $t$ Distributions 35

4.1 Introduction . . . . . . . . . . . . . . . . 35

4.2 The characteristic function of ISP circular model $\ldots \ldots \ldots$

4.3 Skewness . . . . . . . . . . . . . . . . . . . . . . . . . 37

4.3.1 Skewness of Jones and Faddy (JF) ISP skewed- $t$ distribution . 37

4.3.2 Skewness of Azzalini type ISP skewed-t distribution . . . . . 39

4.3.3 Skewness of two-piece ISP skewed- $t$ distribution . . . . . . . 40

5 Estimation of Parameters in ISP Skewed- $t$ Distribution 42

5.1 Introduction . . . . . . . . . . . . . . . . . . . . 42

5.2 Maximum likelihood estimation . . . . . . . . . . . . . . . 42

5.3 Data Analysis . . . . . . . . . . . . . . . . . . . . 43

5.3 .1 Bird migration headings data $\ldots \ldots \ldots \ldots 44$

5.3.2 Drosophila larval locomotion data . . . . . . . . . . . . 48

5.3 .3 Ants data . . . . . . . . . . . . . . . . . . 52

6 Conclusions $\quad 55$

Bibliography . . . . . . . . . . . . . . . . . 64

$\begin{array}{ll}\text { A R-Code } & 65\end{array}$

A.1 R-code for raw circular data plots . . . . . . . . . . 65

A.2 R-code for density plot of ISP skewed- $t$ distributions $\ldots \ldots \ldots 66$

A.3 R-code for maximum likelihood estimation . . . . . . . . 70 
A.4 R-code for histogram with fitted densities . . . . . . . . . . . . . 72 


\section{List of Tables}

5.1 Parameters estimated for Bird migration headings data . . . . . . . . 44

5.2 Comparison of fit for Bird migration headings data . . . . . . . . . 45

5.3 Parameters estimated for mixture distribution for Bird migration headings data. . . . . . . . . . . . . . . . . . 46

5.4 Comparison of fit for mixture distribution for Bird migration headings

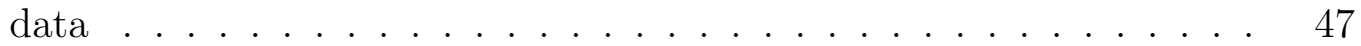

5.5 Parameters estimated for Drosophila larval locomotion data . . . . . 49

5.6 Comparison of fit for Drosophila larval locomotion data . . . . . . . . 49

5.7 Parameters estimated for mixture distribution for Drosophila larval locomotion data . . . . . . . . . . . . . . . . 50

5.8 Comparison of fit for mixture distribution for Drosophila larval locomotion data . . . . . . . . . . . . . . . . . . 51

5.9 Parameters estimated for Ants data . . . . . . . . . . . . . . 53

5.10 Comparison of fit for Ants data . . . . . . . . . . . . 53 


\section{List of Figures}

1.1 Circular data plot of orientations of 100 ants, Fisher 1993, p.243 . . . 4

1.2 Circular data plot of arrival times at an intensive care unit, Fisher

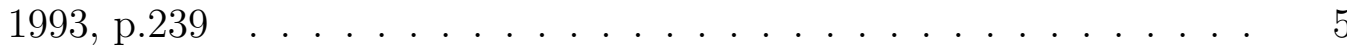

1.3 Histogram of the heading of 1827 migrating birds in Germany, direction measured clockwise from north in radians . . . . . . . . . . . . . 6

1.4 Circular plot of two observation $15^{0}$ and $345^{0} \ldots \ldots$. . . . . . 8

4.1 Probability density functions of Jones and Faddy ISP skewed- $t$ distribution for different values of $a$ and $b \ldots \ldots$. . . . . . . . . . . 38

4.2 Probability density functions of reparametrized JF ISP skewed- $t$ distribution for different values of $\gamma \ldots \ldots 9$

4.3 Probability density functions of Azzalini type ISP skewed- $t$ distribution for different values of $\alpha \ldots \ldots$. . . . . . . . . . . . . . . . . . . . . . . 40

4.4 Probability density functions of two-piece ISP skewed- $t$ distribution for different values of $\gamma \ldots \ldots$. . . . . . . . . . . . . . . 41

5.1 Histogram of the 1827 bird-flight headings together with fitted densities 45

5.2 Histogram of the 1827 bird-flight headings together with fitted mixture densities . . . . . . . . . . . . . . . . . . . . 48

5.3 Histogram of the Drosophila larval locomotion data together with fitted densities . . . . . . . . . . . . . . . . . 50 
5.4 Histogram of the Drosophila larval locomotion data together with fitted mixture densities . . . . . . . . . . . . . . . . . 52

5.5 Histogram of the Ants data together with fitted densities . . . . . 54 


\section{Chapter 1}

\section{Introduction}

\section{$1.1 \quad$ Introduction}

There are various scientific fields, where the observations are 'directions'. For exam-

ple a biologist may be interested in studying the direction of flight of a bird or the orientation of an animal while a geologist may be interested in measuring the direction of earth's magnetic pole. Directional data are often met in Biology, Geography, Geology, Geophysics, Medicine, Meteorology and Oceanography, such as in analysing the origins of comets, solving bird navigational problems, assessing variation in the onset of leukaemia, investigating wind directions etc. There are various statistical problems which arise in the analysis of directional data.

The directions are considered as points on the circumference of a circle in two dimensions or on the surface of a sphere in three dimensions. Generally, directions may be visualized as a points on the surface of hypersphere but observed directions are obviously angular measurements (Mardia, 1975). There have been various successful attempts in modeling directional data, most attention was given to symmetric distributions, and large number of biological data sets were assumed to be symmetric. However, nowadays, interest has increased for skewed distributions to analyse 
data which are clearly not symmetric. This dissertation mainly focuses on asymmetric unimodal circular distributions generated by inverse stereographic projection(ISP) of $\mathbb{R} \rightarrow \mathbb{C}$, a transformation that maps points on the real line to those on a unit circle.

\subsection{Outline of the thesis}

The main purpose of this work is to construct circular models for asymmetric circular data by using Inverse Stereographic Projection. The structure of this thesis is as follows:

- Chapter 1 gives an introduction to circular statistics, review of some literature and parametric circular distributions.

- Chapter 2 discusses different methods of generating circular distributions.

- Chapter 3 introduces three asymmetric circular distributions by using Inverse Stereographic Projection approach on three commonly used skewed- $t$ distributions on the real line.

- Chapter 4 discusses characteristic function and shape characteristics of the proposed distributions.

- Chapter 5 compares the proposed models in terms of their goodness of fit using three data sets that have been previously used.

- Chapter 6 provides conclusions.

\subsection{Circular data and sample statistics}

There are various statistical problems where the data are in the form of angular measurements making orientations or angles in the plane (circular data) or in space 
(spherical data). Circular data is the simple form of directional data, where the single response is not scalar, but angular or directional.The fundamental statistical assumption is that the data are randomly sampled from a population of directions. These observations that can be regarded as points on the circumference of a unit circle or as a unit vector in the plane. This type of data can be transformed to angles by choosing a suitable origin and a sense of rotation (clockwise or counter-clockwise). An important property of this type of data is that different origins or a different sense of rotation produce different values for the same observation. Another important characteristic is periodicity.

Circular data arise in different ways. The two most common sources that rise to circular data correspond to the two principal circular measuring instruments, the compass and the clock. Examples of circular data measured by the compass include wind directions, directions and orientations of birds and animals, ocean current directions and orientation of geological phenomena such as rock cores and fractures. 


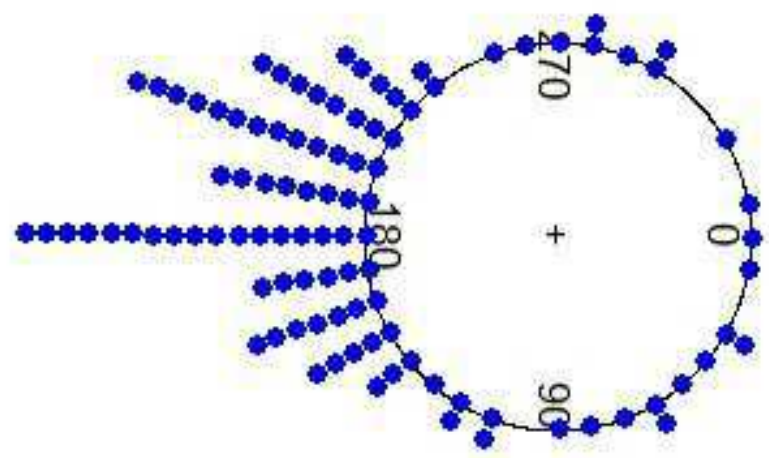

Figure 1.1: Circular data plot of orientations of 100 ants, Fisher 1993, p.243

Figure 1.1 shows the plot of the directions chosen by 100 ants in response to an evenly illuminated black target that was placed at $\pi\left(180^{\circ}\right)$, as an example of circular data obtained using a compass. Most ants tend to find the target, however, few ants miss the target making observations fall around the entire possible range Fisher (1993).

On the other hand, typical data measured by the clock includes arrival times of patients at an intensive care unit in a hospital, incidences of a disease throughout the year, and the number of tourists (daily or monthly) in a city within a year. Similar type of data also arise as times of day (or times of year) of appropriate events (for instance the times of day at which thunderstorms occur), the times of the year at which heavy rain occurs and time of the day a major traffic accident occurs. 


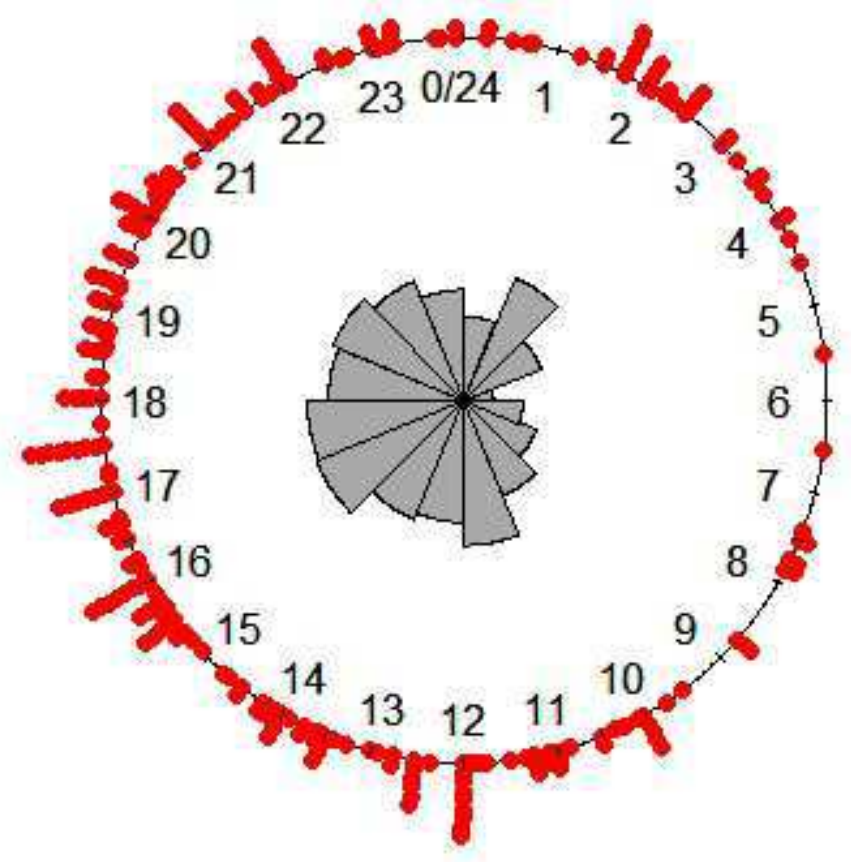

Figure 1.2: Circular data plot of arrival times at an intensive care unit, Fisher 1993, p. 239

An example of such data is presented in Figure 1.2 that shows arrival times on a 24-hour clock of 254 patients at an intensive care unit, over a period of about 12 months, taken from Fisher (1993, p.239). The plots in Figures 1.1 and 1.2 are called rose diagrams. They are better than histograms in depicting the periodic nature of circular data, however histograms are also commonly used.

A histogram of the heading of 1827 migrating birds recorded at an observational post near Stuttgart during the autumnal migration period of 1987, and reported in 
Bruderer and Jenni (1990) is presented in Figure 1.3. Here, the term 'heading' refers to the direction, measured in a clockwise direction from North, of a bird's body during flight. These data will be analyzed in section 5.3.1.

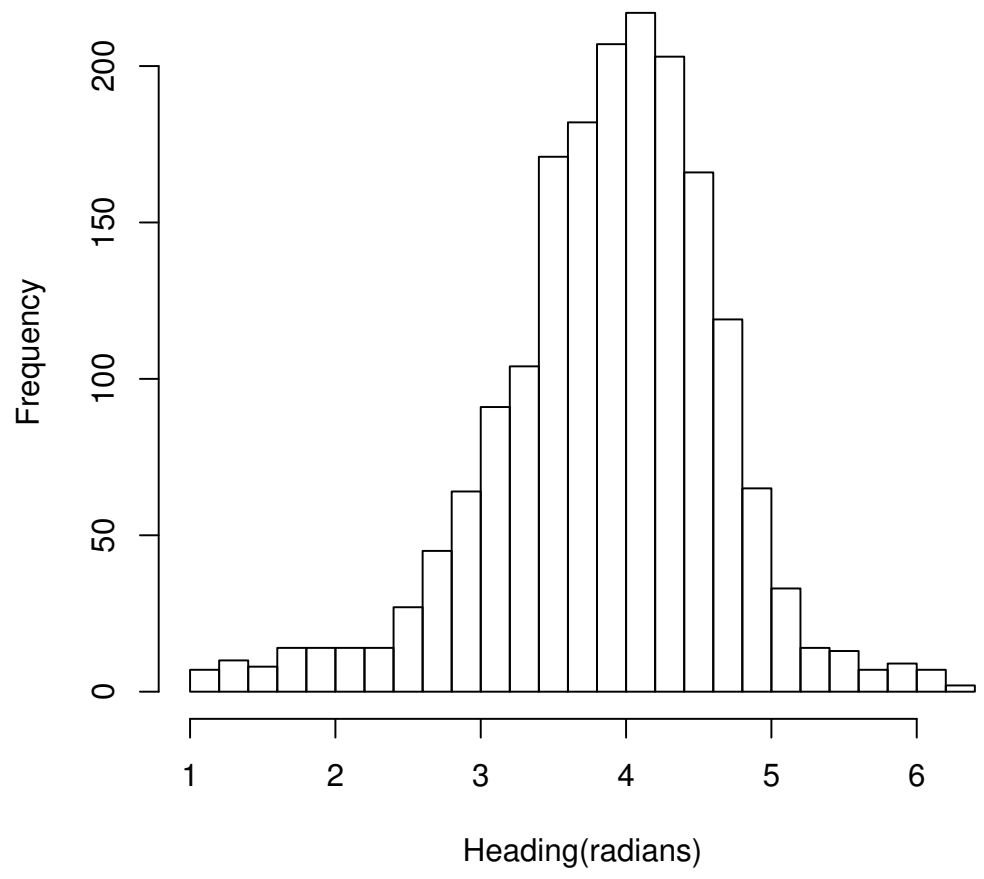

Figure 1.3: Histogram of the heading of 1827 migrating birds in Germany, direction measured clockwise from north in radians

There are two main approaches in directional statistics, namely, the intrinsic approach (directions are considered as points on the circle itself) and the embedding approach (directions are considered as special points in the plane) are commonly used (Mardia and Jupp, 2009). The embedding approach of regarding each point $\theta$ on the circle as the unit vector $X=(\cos \theta, \sin \theta)^{T}$ in the plane enables us to take expectations and thereby define unbiasedness. A single observation $A=\theta^{0}\left(0<\theta \leq 360^{\circ}\right)$ represents the angle made by the vector with positive $\mathrm{X}$-axis (the point $(1,0)$ on the unit circle)in the counter-clockwise direction. The Cartesian co-ordinates of the vec- 
tor are $(x, y)=\left(\cos \theta^{0}, \sin \theta^{0}\right)$.

The important characteristic that differentiates circular data from data measured on a linear scale is its wrap-around nature with no maximum or minimum $(0=2 \pi)$, and in general the measurement is periodic with $\theta$ being the same as $\theta+2 k \pi$ for any integer k. Because of the geometry of the sample space, circular data cannot be modelled using standard statistical techniques. For instance, the sample mean of a data set on the circle (circular mean) is not the usual sample mean (linear mean). To see this, consider a sample of two observations $x_{1}=15^{0}$ and $x_{2}=345^{0}$. Then the linear mean of these two data is simply $180^{\circ}$, but as we can see in Figure 1.4, this value is not a sensible summary of the location of the data. 


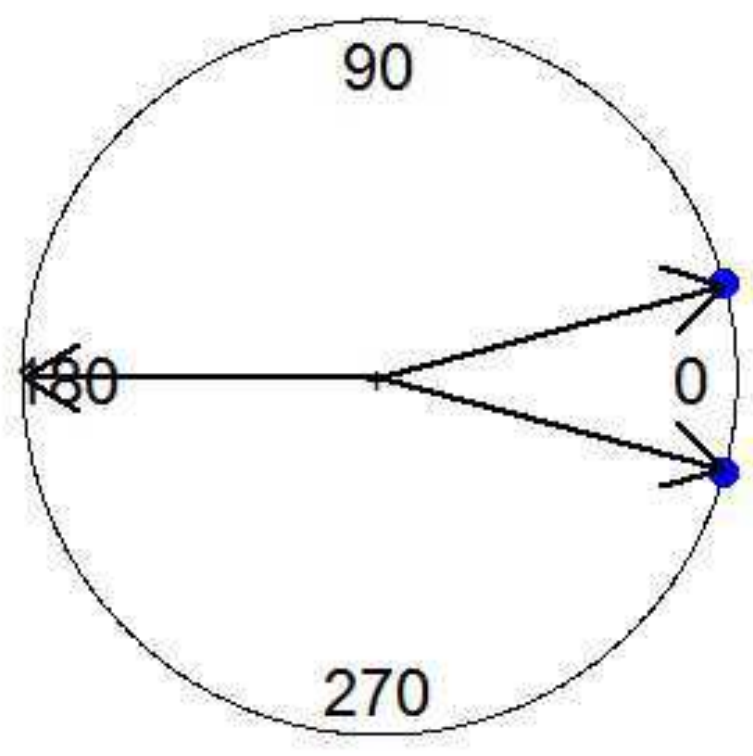

Figure 1.4: Circular plot of two observation $15^{0}$ and $345^{0}$

To calculate the circular mean, one needs to combine all the observations as unit vectors. The normal way to combine unit vectors is vector addition: the direction of the resultant vector will be defined as the mean direction of the individual vectors and the length of the resultant vector will be defined as the mean of the vectors. Suppose a sample is given by $n$ unit vectors $A B_{i}, i=1,2, \ldots, n$, from the center $\mathrm{A}$ of a circle with radius 1 , to points $B_{i}$ on the circumference of the circle. Let $\theta_{i}$ be 
the angular co-ordinate of $A B_{i}$; and let $\bar{S}=\frac{1}{n} \sum_{i=1}^{n} \sin \left(\theta_{i}\right)$ and $\bar{C}=\frac{1}{n} \sum_{i=1}^{n} \cos \left(\theta_{i}\right)$. Then the sample mean direction is defined as

$$
\bar{\theta}=\left\{\begin{array}{l}
\tan ^{-1}\left(\frac{\bar{S}}{\bar{C}}\right), \quad \bar{C} \geqslant 0 \\
\tan ^{-1}\left(\frac{\bar{S}}{\bar{C}}\right)+\pi, \quad \bar{C}<0 .
\end{array}\right.
$$

where the function $\tan ^{-1}()$ is the inverse tangent function which takes values in $\left[-\frac{\pi}{2}, \frac{\pi}{2}\right]$ and the sample mean resultant length is defined as

$$
\bar{R}=\sqrt{\bar{S}^{2}+\bar{C}^{2}}
$$

The size of $\bar{R}$ depends on the variability of the data. If the sample can be regarded as randomly scattered around the circle then $\bar{R} \rightarrow 0$ as $n \rightarrow \infty$ and if the sample is concentrated at $\bar{\theta}$ then $\bar{R} \rightarrow 1$ as $n \rightarrow \infty$.

The $p t h$ sample trigonometric moments about zero direction for $p=1,2, \ldots$ is defined as (Mardia and Jupp, 2009)

$$
m_{p}^{\prime}=a_{p}+i b_{p}
$$

where

$$
a_{p}=\frac{1}{n} \sum_{i=1}^{n} \cos \left(p \theta_{i}\right)
$$

and

$$
b_{p}=\frac{1}{n} \sum_{i=1}^{n} \sin \left(p \theta_{i}\right)
$$

The characteristic function of a circular random variable $\Theta$ is defined as

$$
\phi(t)=E\left(e^{i t \Theta}\right)
$$

and the value of the above function measured at integer $p$ is called the $p t h$ trigonometric moment of $\Theta$

$$
\phi(p)=E\left(e^{i p \Theta}\right)=\int_{0}^{2 \pi} e^{i p \theta} d F(\theta), \quad p= \pm 1, \pm 2, \ldots
$$


By Euler's formula, it follows that

$$
E\left(e^{i p \Theta}\right)=E[\cos (p \Theta)+i \sin (p \Theta)]=\int_{0}^{2 \pi} \cos (p \theta) d F(\theta)+i \int_{0}^{2 \pi} \sin (p \theta) d F(\theta) ;
$$

therefore, the pth trigonometric moment can be written as

$$
\phi(p)=\alpha_{p}+i \beta_{p}
$$

where

$$
\alpha_{p}=E(\cos (p \Theta))=\int_{0}^{2 \pi} \cos (p \theta) d F(\theta)
$$

and

$$
\beta_{p}=E(\sin (p \Theta))=\int_{0}^{2 \pi} \sin (p \theta) d F(\theta)
$$

Here, $\alpha_{p}$ and $\beta_{p}$ are the population analogues for the sample trigonometric moment $a_{p}$ and $b_{p}$. When $p=1$ then $\phi(1)=\alpha_{1}+i \beta_{1}=\rho e^{i \mu}$, where $\mu=\tan ^{-1}\left(\frac{\beta_{1}}{\alpha_{1}}\right)$ is the mean direction and $\rho=\sqrt{\alpha_{1}^{2}+\beta_{1}^{2}}$ is the mean resultant length. And the quantities $\mu$ and $\rho$ are the population analogues for $\bar{\theta}$ and $\bar{R}$. The mean direction $\rho \in[0,1]$, and it characterizes the spread of the distribution.

\subsection{Parametric distributions on circle}

A circular distribution is a probability distribution around a unit circle from 0 to $2 \pi$, which provides corresponding probabilities to different directions. Let $f($.$) be the$ density function of a circular random variable $\Theta$. It satisfies the following conditions

1. $f(\theta) \geqslant 0, \quad$ for $-\infty \leq \theta<\infty$,

2. $\int_{w}^{w+2 \pi} f(\theta) d \theta=1, \quad$ for $\quad 0 \leq w<2 \pi$,

3. $f(\theta)=f(\theta+2 \pi k)$ for any integer $k$

Thus, the density is a periodic, non-negative function with period $2 \pi$ which integrates 
to 1 over any region of length $2 \pi$.

The cumulative distribution function (c.d.f.) $\mathrm{F}$ is defined as

$$
F(w)=P(0 \leq \theta \leq w)=\int_{0}^{w} f(\theta) d(\theta), \quad w \in[0,2 \theta)
$$

and by definition, $F(0)=0$ and $F(2 \pi)=1$.

Now we discuss different circular distributions (Fisher, 1993; Jammalamadaka and SenGupta, 2001).

\subsubsection{The uniform distribution}

Uniform distribution where all directions on the unit circle have equal probability,

that is, there is no preferred direction and the mean direction is undefined. The distribution has the probability density function (p.d.f)

$$
f(\theta)=\frac{1}{2 \pi}, \quad \theta \in[0,2 \pi)
$$

and cumulative distribution function (c.d.f)

$$
F(\theta)=\frac{\theta}{2 \pi}, \quad \theta \in[0,2 \pi)
$$

\subsubsection{The von Mises (VM) distribution}

The von Mises distribution is a symmetric unimodal distribution which is the most commonly used model for symmetric and unimodal samples of circular data. The p.d.f. of the VM distribution is 


$$
f(\theta ; \mu, \kappa)=\frac{1}{2 \pi I_{0}(\kappa)} \exp [\kappa \cos (\theta-\mu)], \quad \theta \in[0,2 \pi), \quad \kappa \in[0, \infty)
$$

where

$$
I_{0}(\kappa)=\frac{1}{2 \pi} \int_{0}^{2 \pi} \exp [\kappa \cos (\phi-\mu)] d \phi
$$

is the modified Bessel function of order zero. The distribution has a maximum value at $\theta=\mu$ and it is symmetric around $\mu$, which is therefore the mean and modal direction. The parameter $\kappa$ is a concentration parameter. As $\kappa \rightarrow 0$, the distribution converges to the uniform distribution; as $\kappa \rightarrow \infty$, the distribution tends to the point distribution concentrated in the direction $\mu$.

\subsubsection{Cardioid distribution}

Perturbation of the uniform density by a cosine function produces the cardioid distribution which has probability density function (Mardia and Jupp, 2009)

$$
f(\theta)=\frac{1}{2 \pi}[1+2 \rho \cos (\theta-\mu)], \quad|\rho|<\frac{1}{2}
$$

The distribution is symmetrical and unimodal with mode at $\mu($ if $\rho>0)$. For $\rho=0$, the cardioid distribution reduces to the uniform distribution.

\subsubsection{Wrapped distribution}

A circular distribution can be obtained by wrapping distributions on the real line around a unit circle. In general, if $X$ is any random variable on the real line with p.d.f. $g(x)$, and c.d.f. $G(x)$, we can obtain circular random variable $\Theta$ by defining: 


$$
\Theta=X[\bmod 2 \pi]
$$

The p.d.f. $f(\theta)$ of $\Theta$ is obtained by wrapping $g(x)$ around the circumference of a circle of unit radius and summing up the overlapping points:

$$
f(\theta)=\sum_{k=-\infty}^{\infty} g(\theta+2 \pi k), \quad \theta \in[0,2 \pi)
$$

with corresponding c.d.f. is

$$
F(\theta)=\sum_{k=-\infty}^{\infty}\{G(\theta+2 \pi k)-G(2 \pi k)\}
$$

\subsubsection{Wrapped Normal (WN) distribution}

The WN distribution is a symmetric unimodal two-parameter distribution which is obtained by wrapping a normal distribution with mean $\mu$ and variance $\sigma^{2}$ around the circle. It arises as the distribution of the location after a fixed time of a particle following a random walk or Brownian motion on the circle (Stephens, 1963). The p.d.f. is

$$
f(\theta ; \mu, \sigma)=\frac{1}{\sigma \sqrt{2 \pi}} \sum_{k=-\infty}^{\infty} \exp \left\{\frac{-(\theta-\mu-2 \pi k)^{2}}{2 \sigma^{2}}\right\}, \quad \theta \in[0,2 \pi) .
$$

The mean resultant length of this distribution is

$$
\rho=e^{-\frac{\sigma^{2}}{2}}
$$

As $\rho \rightarrow 0$, WN tends to the uniform distribution, while as $\rho \rightarrow 1$, it tends to the point distribution concentrated in the direction $\mu$. 


\subsubsection{Wrapped Cauchy (WC) distribution}

The wrapped Cauchy distribution is a symmetric unimodal distribution which is obtained by wrapping a Cauchy distribution around a unit circle. Let X be Cauchy random variable on the real line which has the p.d.f.

$$
f(x ; \mu, \sigma)=\frac{1}{\pi} \frac{\sigma}{\sigma^{2}+(x-\mu)^{2}}, \quad x \in \Re, \quad \sigma>0,-\infty<\mu<\infty .
$$

Then the p.d.f. of wrapped Cauchy distribution is

$$
f(\theta ; \mu, \rho)=\frac{1}{2 \pi} \frac{1-\rho^{2}}{1+\rho^{2}-2 \rho \cos (\theta-\mu)}, \quad 0 \leq \theta<2 \pi
$$

where $\rho=\exp -\sigma$ see Jammalamadaka and SenGupta (2001)

As $\rho \rightarrow 0$, the distribution approaches the uniform distribution and as $\rho \rightarrow 1$, the distribution tends to the point distribution concentrated in the direction $\mu$.

\subsubsection{Wrapped $\mathrm{t}$ (WT) distribution}

Pewsey et al. (2007) introduced the three parameter, symmetric unimodal wrapped $t$ distributions by wrapping a shifted and scaled $t$ distribution onto the unit circle. The wrapped Cauchy distribution discussed in the last section is a special case of wrapped $t$ distributions with the degrees of freedom $(v)$ equals 1.

The p.d.f. of Student- $t$ distribution on the real line is

$$
f(t ; v)=\frac{1}{\sqrt{\nu} \beta\left(\frac{1}{2}, \frac{\nu}{2}\right)}\left(1+\frac{t^{2}}{\nu}\right)^{-\frac{\nu+1}{2}}
$$

where $\nu$ is the degrees of freedom.

Let $Y=\mu+\lambda t$ be the shifted and scaled Student- $t$ random variable. Then wrapping $Y$ around the unit circle, obtained the p.d.f. of the WT distribution as 


$$
f(\theta ; \mu, \lambda, \nu)=\frac{\sqrt{\nu} \beta\left(\frac{1}{2}, \frac{\nu}{2}\right)}{\lambda} \sum_{k=-\infty}^{\infty}\left\{1+\frac{(\theta+2 \pi k-\mu)^{2}}{\lambda^{2} \nu}\right\}^{-\frac{\nu+1}{2}}, \quad \theta \in[0,2 \pi)
$$

The parameters $\nu$ and $\lambda$ define the peakness and the concentration of a WT distribution. When $\nu=1$, then the WT distribution is a wrapped Cauchy (WC)distribution with $\rho=e^{-\lambda}$. It tends to a wrapped normal (WN) distribution when $\nu \rightarrow \infty$.

\subsubsection{Skewed distributions}

As mentioned earlier, skewed circular distributions have become much more of interest in recent years as they are more suitable to model asymmetric circular data. Next, we present some skewed circular distributions recently proposed in the literature.

\subsubsection{Batschelet's distribution}

Batschelet proposed a family of skewed distribution with two parameters. The Batschelet random variable $\Theta$ has the p.d.f.

$$
f(\theta ; \kappa, \nu)=\frac{1}{2 \pi}+\frac{\kappa}{2 \pi} \sin (\theta+\nu \sin \theta), \quad \kappa \in[-1,1], \quad \nu \in[-1,1]
$$

By letting $\Psi=\Theta-\frac{\pi}{2}$, the p.d.f. of the random variable $\Psi$ is

$$
f(\psi ; \kappa, \nu)=\frac{1}{2 \pi}+\frac{\kappa}{2 \pi} \cos (\psi+\nu \cos \psi)
$$

When $\kappa=0$ the distribution tends to a uniform distribution and when $\nu=0$ then it becomes

$$
f(\psi ; \kappa, \nu)=\frac{1}{2 \pi}+\frac{\kappa}{2 \pi} \cos (\psi)
$$


which is a cosine distribution (Fisher, 1993) with mean angle $\mu=0$.

\subsubsection{Wrapped exponential (WE) distribution}

To obtain skewed circular distribution, the principle of wrapping distribution on the real line around the unit circle can be used (Jammalamadaka and Kozubowski, 2001, 2004). The exponential distribution on the real line has p.d.f.

$$
f(x ; \lambda)=\lambda e^{-\lambda x}, \quad x>0
$$

Using the principle of wrapping on the above exponential distribution around the unit circle yields the p.d.f. of the WE distribution

$$
f(\theta ; \lambda)=\frac{\lambda e^{-\lambda \theta}}{1-e^{-2 \pi \lambda}}, \quad \theta \in[0,2 \pi)
$$

When $\lambda=0$, then WE distribution degenerates to the uniform distribution.

\subsubsection{Wrapped skew-Laplace (WSL) distribution}

The skewed Laplace distribution is one of the most common distributions used to describe the logarithm of particle sizes (Fieller et al., 1992), and it has been used to analyze bacterial sizes in axenic cultures (Julia and Vives-Rego, 2005). The distribution has the following density

$$
f(x ; \lambda, \kappa)=\lambda\left(\frac{1}{\kappa}+\kappa\right)^{-1} \begin{cases}e^{-\lambda \kappa|x|}, & x \geqslant 0 \\ e^{-\left(\frac{\lambda}{\kappa}\right)|x|}, & x<0\end{cases}
$$

By using the principle of wrapping the p.d.f. of the WSL distribution Jammalamadaka and Kozubowski (2003) is 


$$
f(\theta ; \lambda, \kappa)=\frac{\lambda \kappa}{1+\kappa^{2}}\left(\frac{e^{-\lambda \kappa \theta}}{1-e^{-2 \pi \lambda \kappa}}+\frac{e^{(\lambda / \kappa) \theta}}{e^{2 \pi \lambda / \kappa}-1}\right), \quad \theta \in[0,2 \pi)
$$

\subsubsection{Wrapped skewed normal (WSN) distribution}

The family of skewed normal (SN) distributions is an extension of the normal family obtained by adding s shape parameter to determine skewness. The skewed normal distribution on the real line was proposed by (Azzalini, 1985). Let the linear random variable $\mathrm{X}$ be skewed normal with skewness parameter $\lambda$. Then the standard skewed normal density with shape parameter $\lambda$ is

$$
f(x ; \lambda)=2 \phi(x) \Phi(\lambda x), \quad-\infty<x<\infty, \quad-\infty<\lambda<\infty
$$

where $\phi($.$) and \Phi($.$) are the standard normal density and distribution functions re-$ spectively. Considering location parameter $\xi$ and scale parameter $\eta$, let $Y=\xi+\eta X$ and the random variable $\mathrm{Y}$ has the density

$$
f(y ; \xi, \lambda, \eta)=\frac{2}{\eta} \phi\left(\frac{y-\xi}{\eta}\right) \Phi\left\{\lambda\left(\frac{y-\xi}{\eta}\right)\right\}
$$

where $-\infty<y<\infty,-\infty<\xi<\infty, \eta>0,-\infty<\lambda<\infty$. The characteristic function of $Y$ was proposed by (Azzalini and Capitanio, 1999). Pewsey (2006, 2000) studied the WSN distributions and suggested the characteristic function for these distributions.

Using the principle of wrapping, the circular random variable $\Theta=\mathrm{Y}(\bmod 2 \pi)$, corresponding to wrapping $\mathrm{Y}$ onto the unit circle, has density

$$
f(\theta ; \xi, \lambda, \eta)=\frac{2}{\eta} \sum_{k=-\infty}^{\infty} \phi\left(\frac{\theta+2 \pi k-\xi}{\eta}\right) \Phi\left\{\lambda\left(\frac{\theta+2 \pi k-\xi}{\eta}\right)\right\}, \quad \theta \in[0,2 \pi)
$$

As $\eta \rightarrow 0$, the distribution approaches to a point distribution, as $\eta \rightarrow \infty$, the distri- 
bution tends to the uniform distribution and when $\lambda=0$, the distribution becomes a WN distribution.

\subsection{Literature review}

Research on directional data can be traced back to the 18th century. Rayleigh (1880) studied the distribution of the resultant length of normal vectors and developed Rayleigh's one sample test. Exact and approximate tests for mean direction is ex-

plained by Stephens (1962) and also worked on Random walk on a circle in Stephens (1963). Mardia (1975) discussed statistics of directional data. Mardia and Sutton (1975) have worked on the modes of a mixture of two Von Mises distributions. Beran (1979) has worked on Exponential models for directional data. A characterization of uniform distribution on the circle is studied by Kent et al. (1979). Batschelet (1981) introduced an influential book on circular statistics discussing applications in Biology. Some statistical methods for bivariate circular data has been studied by Rivest (1982). Fisher (1993) Fisher's (1993) book presents material for analysing circular data along with many real life examples that has become an important source of reference in dealing with circular data. Jammalamadaka and SenGupta (1998) have worked on predictive inference for directional data. Pewsey (2000) introduced the wrapped skew-normal distribution on the circle.

Jammalamadaka and Kozubowski (2001) have developed a wrapped exponential circular model. Pewsey (2002) contributed more on testing circular symmetry. A new family of circular models: the wrapped Laplace distributions was introduced by Jammalamadaka and Kozubowski (2003). Fernández-Durán (2004) have introduced a new family of circular distributions based on nonnegative trigonometric sums and extended their work Fernández-Durán (2007) in constructing bivariate models for circular-circular and circular-linear data. Jones and Pewsey (2005) have worked on a family of symmetric distributions on the circle. Arnold and SenGupta (2006) have 
proposed a method on probability distributions and statistical inference for axial data. An asymmetric circular-linear multivariate regression models with applications to environmental data was given by SenGupta and Ugwuowo (2006). Dattatreya Rao et al. (2007) proposed the following new wrapped models (i) Wrapped Lognormal (ii) Wrapped Logistic (iii) Wrapped Weibull and (iv) Wrapped Extreme Value Distributions.

Pewsey et al. (2007) introduced the wrapped $t$ family of circular distributions. The wrapped stable family of distributions as a flexible model for circular data was given by Pewsey (2008). Sarma et al. (2009) studied characteristic function of Wrapped Half Logistic and Wrapped Binormal Distribution. Abe et al. (2009) studied on Papakonstantinou's extension of the cardioid distribution and also derived symmetric unimodal models for directional data motivated by inverse stereographic projections Abe et al. (2010). Kato and Jones (2010) discussed a family of distributions on the circle with links to, and applications arising from, Möbius transformation. Characteristic functions of the wrapped lognormal and the wrapped Weibull distributions was derived by Sarma et al. (2011). A sine-skewed circular distributions proposed by Abe and Pewsey (2011).

Dattatreya Rao et al. (2011) introduced Stereographic Logistic model and Stereographic Lognormal Distribution was given by Girija et al. (2013b). Jones and Pewsey (2012) studied an inverse Batschelet distributions for circular data. Dattatreya Rao et al. (2013) discussed on The Rising Sun Wrapped Lognormal and The Rising Sun Wrapped Exponential Models. Also Radhika et al. (2013) studied on rising Sun von mises and rising Sun wrapped cauchy circular models. Kato et al. (2013) introduced an extended family of circular distributions related to wrapped Cauchy distributions via Brownian motion. Girija et al. (2013a) proposed on Bimodal Offset Cauchy Distribution and a New Circular Model Induced by Inverse Stereographic Projection on Double Exponential Model-Application to Birds Migration Data in 
Girija et al. (2014). Yedlapalli et al. (2014) studied a new circular model induced by modified Inverse Stereographic Projection on arctan exponential-type distribution. Kato and Jones (2015) discussed a tractable and interpretable four-parameter family of unimodal distributions on the circle. Kato and Pewsey (2015) studied a Möbius transformation-induced distribution on the torus. Chaubey and Midhu (2015) discussed circular distributions arising from the Möbius transformation of wrapped distributions similar to those studied in Kato and Jones (2010). Smooth kernel estimation of a circular density was addressed by Fisher (1989) using symmetric kernels. Recently Di Marzio et al. (2009) and Taylor (2008) have considered kernel estimation using the circular kernels. Chaubey (2016) has provided a connection to orthogonal polynomials on the unit circle and kernel estimation using wrapped Cauchy distribution. 


\section{Chapter 2}

\section{Generation of Circular}

\section{Distributions}

\section{$2.1 \quad$ Introduction}

A circular random variable can be represented either in terms of the angle $\theta, \quad(0 \leq$ $\theta<2 \pi)$ or as the two-dimensional unit vector $(X=\cos \theta, Y=\sin \theta)^{\prime}$. In general a probability model defined on the real line may be transformed to a circular distribution by proper transformation. We have seen an example of this in the previous chapter as the circular uniform distribution that is basically obtained by mapping points from $[0,1]$ to $[0,2 \pi]$. In such distributions, periodicity is implicitly assumed. In this chapter we will discuss several methods of obtaining circular distribution. For further discussion in this thesis, one of the methods called the inverse stereographic projection (ISP) method will be considered in detail.

\subsection{Methods of generating circular distributions}

A large number of important and interesting circular models may be generated from different known probability distributions on the real line or on the plane, by a va- 
riety of mechanisms. We discuss four such general methods (Jammalamadaka and SenGupta, 2001):

(1) Wrapping : Wrapped circular distributions can be obtained by wrapping a linear distribution around the unit circle. Any linear random variable $\mathrm{X}$ on the real line may be transformed to a circular random variable by reducing its modulo $2 \pi$ i.e., using

$$
\theta=X(\bmod 2 \pi)
$$

This process corresponds to taking the real line and wrapping it around the circle of unit radius, accumulating probability over all the overlapping points $x=$ $\theta, \theta \pm 2 \pi, \theta \pm 4 \pi, \ldots$. This is clearly a many-to-one mapping so that if $f(x)$ is the density of the real-valued random variable and $g(\theta)$ represents the circular density, we have

$$
g(\theta)=\sum_{k=-\infty}^{\infty} f(\theta+2 \pi k), \quad \theta \in[0,2 \pi)
$$

By this approach, both discrete and continuous wrapped distributions may be constructed. Few wrapped distributions are discussed in the last chapter.

(2) Conditioning/Characterizing : By characterizing properties such as maximum entropy, it is more informative to ask if there are distributions on the circle which enjoy certain desirable properties. For example, one may ask which distribution has the maximum entropy subject to having non-zero first trigonometric moment. The uniform and von Mises (circular normal) distributions have the maximum entropy (Mardia and Jupp, 2009) where the entropy of a distribution on the circle with probability density function $f$ is defined as 


$$
H(f)=-\int_{0}^{2 \pi} f(\theta) \log f(\theta) d \theta .
$$

This is one way of measuring the closeness of a distribution to the uniform distribution. In addition, if we ask which distribution on the circle has the property that the sample mean direction and the length of the resultant vector are independent, then the uniform or isotropic distribution is the answer (Kent et al., 1979; Jammalamadaka and SenGupta, 2001). This characterization of the uniform distribution is similar to and as useful as that of the normal distribution on the real line as the one for which the sample mean and sample variance are independent.

Suppose the direction of the sample resultant vector $\overline{\alpha_{0}}$ provides a reasonable mean direction for a given sample. Suppose one wants to characterize a circular distribution such that the sample mean direction has the maximum probability around the population mean direction, then according to von Mises (1918), the circular normal distribution has this property. The proof is simple and is reproduced below.

Let $\alpha_{1}, \alpha_{2}, \ldots, \alpha_{n}$ be the observations from the density $f(\alpha-\gamma)$, where $\gamma$ may represent the mean direction. Now the likelihood is given by

$$
L=\prod_{i=1}^{n} f\left(\alpha_{i}-\gamma\right)
$$

and we obtain the likelihood equation

$$
\frac{\partial \log L}{\partial \gamma}=\text { const. } \sum_{i=1}^{n} \frac{f^{\prime}\left(\alpha_{i}-\gamma\right)}{f\left(\alpha_{i}-\gamma\right)}=0
$$

On the other hand, if $\gamma$ were to be estimated by $\overline{\alpha_{0}}$, we also have

$$
\sum_{i=1}^{n} \sin \left(\alpha_{i}-\gamma\right)=0
$$


Equations (2.1) and (2.2) hold for all arbitrary $\alpha_{i}$ and all $n$, so the equality must hold term by term and thus,

$$
\frac{f^{\prime}(\alpha-\gamma)}{f(\alpha-\gamma)}=\kappa \sin (\alpha-\gamma)
$$

for some constant $\kappa$. Hence,

$$
f(\alpha-\gamma)=c e^{\kappa \cos (\alpha-\gamma)}
$$

which gives us the circular normal density.

(3) Offsetting : Offset distribution is a distribution on the plane which is obtained by transforming a bivariate linear random variable to just its directional component. This is constructed by accumulating probabilities over all different lengths for a given direction. We transform the bivariate random vector $(\mathrm{X}, \mathrm{Y})$ into polar co-ordinates $(r, \theta)$ and integrate over $r$ for a given $\theta$. If $f(x, y)$ denotes the joint distribution of a bivariate distribution on the plane, the resulting circular offset distribution, say $g(\theta)$, is written by

$$
g(\theta)=\int_{0}^{\infty} f(r \cos \theta, r \sin \theta) r d r
$$

For instance, the offset normal $(\mathrm{ON})$ distribution is constructed from the bivariate normal distribution $\phi(x, y ; \mu, \Sigma)$ with mean $\mu=\left(\mu_{1}, \mu_{2}\right)^{\prime}$ and covariance matrix $\Sigma$. If $\rho$ denotes the correlation between the variables and $\sigma_{1}^{2}, \sigma_{2}^{2}$ their variances, the probability density function of the offset normal distribution is given by

$$
f(\theta)=\frac{1}{C(\theta)}\left\{\phi(\mu, \nu ; 0, \Sigma)+a D(\theta) \Phi[D(\theta)] \phi\left[\frac{a(\mu \sin \theta-\nu \cos \theta)}{\sqrt{C(\theta)}}\right]\right\}
$$

where

$$
\begin{gathered}
a=\frac{1}{\sigma_{1} \sigma_{2} \sqrt{1-\rho^{2}}} \\
C(\theta)=a^{2}\left(\sigma_{2}^{2} \cos ^{2}(\theta)-\rho \sigma_{1} \sigma_{2} \sin 2 \theta+\sigma_{1}^{2} \sin ^{2} \theta\right),
\end{gathered}
$$




$$
D(\theta)=\frac{a^{2}}{\sqrt{C(\theta)}}\left[\mu \sigma_{2}\left(\sigma_{2} \cos \theta-\rho \sigma_{1} \sin \theta\right)+\nu \sigma_{1}\left(\sigma_{1} \sin \theta-\rho \sigma_{2} \cos \theta\right)\right]
$$

and $\phi(),. \Phi($.$) are the pdf and cdf of N(0,1)$ respectively.

The particular case when $\mu=0$ and $\rho=0$ we obtain the density function as

$$
f(\theta)=\frac{\sqrt{1-b^{2}}}{2 \pi(1-b \cos 2 \theta)}
$$

where

$$
b=\frac{\sigma_{1}^{2}-\sigma_{2}^{2}}{\sigma_{1}^{2}-\sigma_{2}^{2}}
$$

This has been widely used by meteorologists for wind direction under the assumption that the $X$ and $Y$ components of the wind vector are independently distributed as $N\left(0, \sigma_{1}^{2}\right)$ and $N\left(0, \sigma_{2}^{2}\right)$ respectively.

(4) Inverse Stereographic Projection : One may start with a distribution on the real line $\mathbb{R}$, and apply a stereographic projection that identifies points $x$ on $\mathbb{R}$ with those on the circumference of the circle, say $\theta$. This correspondence is one-toone except for the fact that the mass if any, at both $+\infty$ and $-\infty$, are identified with $\pi$. Moreover, Inverse Stereographic Projection (ISP) is defined by a one-to-one mapping given by

$$
T(\theta)=x=u+v \frac{\sin \theta}{1+\cos \theta}=u+v \tan \left(\frac{\theta}{2}\right)
$$

where $x \in(-\infty, \infty), \theta \in[-\pi, \pi), u \in \mathbb{R}$, and $v>0$. Then by Minh and Farnum $(2003)$

$$
T^{-1}(x)=\theta=2 \tan ^{-1}\left\{\frac{(x-u)}{v}\right\}
$$

is a random point on the unit circle.

Let $f(x)$ and $F(x)$ be the density function and distribution function of the random variable $X$ respectively. Also $g(\theta)$ and $G(\theta)$ denote the density function and distribution function of the random point $\theta$ on the unit circle respectively. Then $G(\theta)$ and 
$g(\theta)$ can be written in terms of $F(x)$ and $f(x)$ using the following equations as stated below.

For $v>0$,

$$
\begin{array}{r}
G(\theta)=F\left(u+v \tan \left(\frac{\theta}{2}\right)\right)=F(T(\theta)) \\
g(\theta)=\frac{v}{2}\left(1+\tan ^{2}\left(\frac{\theta}{2}\right)\right) f\left(u+v \tan \left(\frac{\theta}{2}\right)\right) \\
=\frac{v}{2}\left(1+\left(\frac{T(\theta)-u}{v}\right)^{2}\right) f(T(\theta))
\end{array}
$$




\section{Chapter 3}

\section{Inverse Stereographic Projection Circular Skewed- $t$ Distributions}

\subsection{Introduction}

Student's $t$-distribution is well known in Statistics. It was introduced as normality based sampling distribution and it has enjoyed wide applications over the years. However, beside its ubiquitous use as a sampling distribution, it now figures widely as an empirical model for heavy-tailed data, particularly in finance, see (Rachev et al., 2005). Recently, there has been much interest in the modelling of asymmetry together with heavy tails. Inverse stereographic projection method of generating circular distribution is discussed in the previous chapter. In this chapter we will discuss three different skewed- $t$ distributions on the real line and apply inverse stereographic projection method to obtain three asymmetric circular distributions which is the main interest of this thesis. An asymmetric distribution in this fashion will be called ISP circular skewed- $t$ distribution. 


\subsection{Skewed- $t$ distribution on the real line}

Sometimes, it is useful to consider an alternative to the normal or $t$-distribution which is both heavy tailed and skewed. There are different skewed-t distributions which were proposed by different authors following different techniques. Amongst these the following distributions figure promptly: the skewed- $t$ distribution of Jones and Faddy (2003), obtained by transforming a beta random variable; the skewed- $t$ distribution based on Azzalini (1985) general form of creating skewed distributions that is obtained by a specific weighting function (Rosco et al., 2011; Ma and Genton, 2004; Genton, 2004; Sahu et al., 2003; Azzalini and Capitanio, 2003; Branco and Dey, 2001) , the two-piece skewed- $t$ distribution (Rosco et al., 2011; Ferreira and Steel, 2007; Fernández and Steel, 1998). A brief discussion of these three skewed-t distribution are given below.

\subsubsection{The Jones and Faddy (2003) skewed- $t$ distribution}

Jones and Faddy proposed a family of distributions which includes the symmetric t-

distribution as special cases, and also includes extension of the t-distribution, taking value on the real line, with non-zero skewness. Jones and Faddy (2003) introduced the skewed- $t$ family with density

$$
f_{J F}(x ; a, b)=\frac{1}{\beta(a, b) 2^{a+b-1} \sqrt{a+b}}\left(1+\frac{x}{\sqrt{a+b+x^{2}}}\right)^{a+\frac{1}{2}}\left(1-\frac{x}{\sqrt{a+b+x^{2}}}\right)^{b+\frac{1}{2}}
$$

where $a>0$ and $b>0$ be parameters and $\beta(.,$.$) is the beta function. When a=b$ then $f$ reduces to the $t$-distribution on $2 a$ degrees of freedom. When $a>b$ or $a<b$, $f$ is positively or negatively skewed respectively. In reality, $f(x ; a, b)=f(-x ; a, b)$. Also note that $\mathrm{a}$ and $\mathrm{b}$ are positive real numbers and need not be integer or half integer.

Details including two derivations of this distribution is given in Jones (2001). The first is a mathematical manipulation in which the symmetric $t$-density function is 
factorized into two parts and those parts are taken to different powers. Though one important application of the skewed- $t$ distribution is in robustness studies, it is the significant robust data modelling aspect of the skewed- $t$ distribution, as a model for data coming from a skewed and/or heavy-tail distribution.

\subsubsection{The Azzalini skewed- $t$ distribution}

The Azzalini (1985) type skewed- $t$ family is obtained from a general formula for constructing skewed distributions from a symmetric distribution as given by

$$
f(x ; \alpha)=2 g(x) G(\alpha x), \quad x, \alpha \in \mathbb{R},
$$

where $g$ and $G$ are the density and distribution functions, respectively, of a symmetric distribution. When $g$ is the standard normal density $\phi$ then $f$ provides the well-known skew-normal distribution. The parameter $\alpha$ is the skewness parameter, with positive and negative $\alpha$ leading to positive and negative skewness respectively. Also $\alpha=0$ corresponds to symmetric density $g$. A lot of extensions exist, replacing $G(\alpha x)$ in (3.2) by a host of other skewing functions (see Jones, 2008).

The density of the Azzalini type skewed- $t$ distribution, considered in Rosco et al. (2011), is obtained by replacing $g$ and $G$ in equation (??) by $g_{\nu}$, and $G$, respectively that gives

$$
f_{A}(x ; \nu, \alpha)=2 g_{\nu}(x) G_{\nu+1}\left(\alpha x \sqrt{\frac{\nu+1}{\nu+x^{2}}}\right), \quad x, \alpha \in \mathbb{R}
$$

\subsubsection{The two-piece skewed- $t$ distribution}

An alternative type of the skewed- $t$ family comprises 'two-piece' distributions made

up of differently scaled halves of a symmetric distribution. In general, the two-piece $t$ distribution is given by

$$
f_{T P}(x ; \nu, \gamma)=f_{\nu}\left(\frac{x}{1+\gamma}\right) I(x<0)+f_{\nu}\left(\frac{x}{1-\gamma}\right) I(x \geq 0),
$$


where $I$ denotes the indicator function and $-1<\gamma<1$ and $f_{\nu}$ denote the density functions of the $t$ distribution on $\nu$ degrees of freedom, denoted $t_{\nu}$. Although various equivalent parametrizations exist, (see Rosco et al., 2011; Jones, 2006). The parameter $\gamma$ is a skewness parameter, with positive and negative $\gamma$ leading to negative and positive skewness and $\gamma=0$ corresponds to symmetric density.

\subsection{Inverse Stereographic Projection (ISP) skewed- $t$ distribution}

By applying ISP method defined by a one to one mapping in equation (2.3) in the three different skewed- $t$ distribution on the real line discussed in the previous section we will obtain three different circular ISP skewed- $t$ distribution. In the later sections we will discuss in details about these circular ISP skewed- $t$ distribution.

\subsubsection{Jones and Faddy ISP skewed- $t$ distribution}

Considering the Inverse Stereographic Projection (2.3) of the Jones and Faddy skewed$t$ distribution (3.1), the corresponding circular p.d.f. by using equation (2.5) is given by

$$
g_{J F}(\theta ; a, b, v)=\frac{v\left(1+\tan ^{2}\left(\frac{\theta}{2}\right)\right)}{2^{m} \beta(a, b) \sqrt{m}}(1+\Psi(\theta))^{a+\frac{1}{2}}(1-\Psi(\theta))^{b+\frac{1}{2}}
$$

where $u=0, v>0,-\pi \leq \theta<\pi$ and $m=a+b$ and

$$
\Psi(\theta)=\frac{v \tan \left(\frac{\theta}{2}\right)}{\sqrt{m+v^{2} \tan ^{2}\left(\frac{\theta}{2}\right)}}
$$

For $a=b=\frac{m}{2}$, using the identity

$$
\frac{1}{1+x^{2}}=1-\frac{x^{2}}{1+x^{2}}=\left(1+\frac{x}{\sqrt{1+x^{2}}}\right)\left(1-\frac{x}{\sqrt{1+x^{2}}}\right)
$$


and the Legendre duplication formula for the Gamma function,

$$
\Gamma(2 a) \sqrt{2 \pi}=2^{2 a-\frac{1}{2}} \Gamma(a) \Gamma\left(a+\frac{1}{2}\right)
$$

the equation (3.5) reduces to the modified Minh-Farnum symmetric circular distribution (Abe et al., 2010) given as

$$
f_{M M F}(\theta)=\frac{v}{2 \sqrt{m} \beta\left(\frac{m}{2}, \frac{1}{2}\right)} \frac{\left(1+\tan ^{2}\left(\frac{\theta}{2}\right)\right)}{\left(1+\frac{v^{2} \tan ^{2}\left(\frac{\theta}{2}\right)}{m}\right)^{\frac{(m+1)}{2}}}
$$

The skewed version of the Cartwrite's power-of-cosine pdf is obtained by substituting $v=\sqrt{m}$ in equation (3.5), namely

$$
f(\theta ; a, b)=\frac{1}{2^{m} \beta(a, b)}\left(1+\sin \left(\frac{\theta}{2}\right)\right)^{a-\frac{1}{2}}\left(1+\sin \left(\frac{\theta}{2}\right)\right)^{b-\frac{1}{2}}
$$

Both of these versions can adapt the additional location parameter $\mu$ that we omit for simplicity. Wang and Shimizu (2012) and other types of skewed- $t$ densities such that one by Azzalini and Capitanio (2003) may also be used.

An alternative form of given density in equation (3.5) can be written as

$$
g_{J F}(\theta ; a, b, v)=\frac{v_{0}}{2^{m} \beta(a, b)} \frac{2}{1+\cos \theta}(1+\Psi(\theta))^{a+\frac{1}{2}}(1-\Psi(\theta))^{b+\frac{1}{2}}
$$

where, $a+b=m, v_{0}=\frac{v}{\sqrt{m}}$ and $\Psi(\theta)=\frac{v_{0} \sin \theta}{\sqrt{(1+\cos \theta)^{2}+v_{0}^{2} \sin ^{2} \theta}} \quad$ and $-\pi \leq \theta<\pi$. The proof of the form (3.8) is given below:

\section{Proof.}

$$
\text { First, } \quad 1+\tan ^{2}\left(\frac{\theta}{2}\right)=\sec ^{2}\left(\frac{\theta}{2}\right)=\frac{1}{\cos ^{2}\left(\frac{\theta}{2}\right)}=\frac{2}{1+\cos \theta}
$$




$$
\begin{aligned}
& \text { Second, } \quad \Psi(\theta)=\frac{v \tan \left(\frac{\theta}{2}\right)}{\sqrt{m+v^{2} \tan ^{2}\left(\frac{\theta}{2}\right)}} \\
& =\frac{v_{0} \tan \left(\frac{\theta}{2}\right)}{\sqrt{1+v_{0}^{2} \tan ^{2}\left(\frac{\theta}{2}\right)}} \quad \text { where, } v_{0}=\frac{v}{\sqrt{m}} \\
& =\frac{v_{0} \sqrt{\sec ^{2}\left(\frac{\theta}{2}\right)-1}}{\sqrt{1+v_{0}^{2}\left(\sec ^{2}\left(\frac{\theta}{2}\right)-1\right)}} \\
& =\frac{v_{0} \sqrt{1-\cos ^{2}\left(\frac{\theta}{2}\right)}}{\sqrt{v_{0}^{2}+\left(1-v_{0}^{2}\right) \cos ^{2}\left(\frac{\theta}{2}\right)}} \\
& =\frac{v_{0} \sqrt{1-\frac{1}{2}(1+\cos \theta)}}{v_{0}^{2}+\frac{1}{2}\left(1-v_{0}^{2}\right)(1+\cos \theta)} \\
& =\frac{v_{0} \sqrt{1-\cos \theta}}{\sqrt{1+v_{0}^{2}+\left(1-v_{0}^{2}\right) \cos \theta}} \\
& =\frac{v_{0} \sin \theta}{\sqrt{\left(1+v_{0}^{2}+\left(1-v_{0}^{2}\right) \cos \theta\right)(1+\cos \theta)}} \\
& =\frac{v_{0} \sin \theta}{\sqrt{v_{0}^{2}\left(1-\cos ^{2} \theta\right)+1+2 \cos \theta+\cos ^{2} \theta}} \\
& =\frac{v_{0} \sin \theta}{\sqrt{(1+\cos \theta)^{2}+v_{0}^{2} \sin ^{2} \theta}} \text {. }
\end{aligned}
$$

\subsubsection{Reparametrization of Jones and Faddy ISP skewed- $t$ distribution}

Reparametrization does not produce a different distribution family but simply reexpresses the original parameters into new ones, so that these can be better interpreted (Jones, 2006). The skewness on the circle is naturally defined to have the opposite sign from the skewness of its usual linear representation (Jones and Pewsey, 2012). For Jones and Faddy ISP skewed-t family, skewness depends on both the parameters $a$ and $b$, through the ratio $a / b$. Hence we define the new parameters in the case of Jones and Faddy ISP skewed- $t$ family as: 


$$
\gamma=\frac{a}{b} \text { and } m=a+b
$$

Hence the reparametrized form of the Jones and Faddy ISP skewed- $t$ family is obtained from Eq (3.5) by choosing

$$
a=\frac{m \gamma}{1+\gamma} \text { and } b=\frac{m}{1+\gamma}
$$

The p.d.f. of the reparametrized Jones and Faddy skewed- $t$ is

$$
g_{J F}(\theta ; m, v, \gamma)=\frac{v\left(1+\tan ^{2}\left(\frac{\theta}{2}\right)\right)}{2^{m} \beta\left(\frac{m \gamma}{1+\gamma}, \frac{m}{1+\gamma}\right) \sqrt{m}}(1+\Psi(\theta))^{\frac{m \gamma}{1+\gamma}+\frac{1}{2}}(1-\Psi(\theta))^{\frac{m}{1+\gamma}+\frac{1}{2}}, \quad v, m, \gamma>0
$$

where,

$$
\Psi(\theta)=\frac{v \tan \left(\frac{\theta}{2}\right)}{\sqrt{\left(m+v^{2} \tan ^{2}\left(\frac{\theta}{2}\right)\right)}} \text { and }-\pi \leq \theta<\pi
$$

Here $\gamma$ is interpreted as the skewness parameter; $\gamma>1$ leads to positive skewness and $\gamma<1$ leads to negative skewness, where as $\gamma=1$ corresponds to a symmetric density.

\subsubsection{Azzalini type ISP skewed- $t$ distribution}

By applying the Inverse Stereographic Projection (2.3) on the Azzalini type skewed- $t$ distribution (3.3), the pdf of the circular Azzalini type ISP skewed- $t$ distribution by using equation (2.5) is given by

$$
g_{A}(\theta ; \nu, v, \alpha)=\frac{v\left(1+\tan ^{2}\left(\frac{\theta}{2}\right)\right)}{\sqrt{\nu} \beta\left(\frac{\nu}{2}, \frac{1}{2}\right)} \frac{1}{\left(1+\frac{v^{2} \tan ^{2}\left(\frac{\theta}{2}\right)}{\nu}\right)^{\frac{\nu+1}{2}}}\left[1-\frac{1}{2} I\left(Z(\theta), \frac{\nu+1}{2}, \frac{1}{2}\right)\right]
$$


where

$$
Z(\theta)=\frac{(\nu+1)\left(\nu+v^{2} \tan ^{2}\left(\frac{\theta}{2}\right)\right)}{\nu^{2}+v^{2} \tan ^{2}\left(\frac{\theta}{2}\right)\left(\nu+\alpha^{2}(\nu+1)\right)}
$$

and $-\pi \leq \theta<\pi, \alpha \in \mathbb{R}, \mathrm{I}$ is the regularized incomplete beta function (Johnson et al., 1995), $v>0$ and $\nu$ is the degrees of freedom. The parameter $\alpha$ controls skewness and positive and negative $\alpha$ leading to positive and negative skewness respectively. Also $\alpha=0$ corresponds to symmetric density.

\subsubsection{Two-piece ISP skewed- $t$ distribution}

By using the Inverse Stereographic Projection (2.3) on the two-piece skewed- $t$ distribution (3.4), the pdf of the circular two-piece ISP skewed- $t$ distribution by using equation (2.5) is given by

$g_{T P}(\theta ; \nu, v, \gamma)=\frac{v\left(1+\tan ^{2}\left(\frac{\theta}{2}\right)\right)}{2 \sqrt{\nu} \beta\left(\frac{\nu}{2}, \frac{1}{2}\right)}\left[\frac{1}{\left(1+\kappa_{1}(\theta, \gamma)\right)^{\frac{\nu+1}{2}}} I(\theta<0)+\frac{1}{\left(1+\kappa_{2}(\theta, \gamma)\right)^{\frac{\nu+1}{2}}} I(\theta \geq 0)\right]$

where, $\kappa_{1}(\theta, \gamma)=\frac{v^{2} \tan ^{2}\left(\frac{\theta}{2}\right)}{\nu(1+\gamma)^{2}}, \kappa_{2}(\theta, \gamma)=\frac{v^{2} \tan ^{2}\left(\frac{\theta}{2}\right)}{\nu(1-\gamma)^{2}},-\pi \leq \theta<\pi, v>0,-1<$ $\gamma<1, \nu$ is the degrees of freedom and I is the indicator function. The parameter $\gamma$ controls skewness and negative and positive $\gamma$ leading to the positive and negative skewness respectively. Also $\gamma=0$ corresponds to symmetric density. 


\section{Chapter 4}

\section{Shape Characteristics of ISP Skewed- $t$ Distributions}

\subsection{Introduction}

Estimation of moments in the case of data on real line is replaced by estimation of the trigonometric moments in the case of circular data. The characteristic functions corresponding to the newly constructed ISP circular distributions are not explicitly available, hence they can be evaluated using numerical methods. In this chapter we will discuss the way of calculating characteristic functions of the new circular models and present the graphs of the corresponding moments.

\subsection{The characteristic function of ISP circular model}

The characteristic function of a circular model with probability density function $g(\theta)$ is defined as

$$
\phi_{\theta}(p)=\int_{0}^{2 \pi} e^{i p \theta} g(\theta) d \theta, p \in \mathbb{Z} .
$$

Sarma et al. $(2011,2009)$ developed the characteristic functions of some new wrapped models based on the result given in Jammalamadaka and SenGupta (2001). This 
result can not be applied directly in case of ISP circular model. The characteristic function of an ISP circular model can be obtained in terms of respective linear model (Girija et al., 2014). The following theorem related to the characteristic function of linear model which is applied here in the case of ISP circular models was established by Lukacs (1970).

Theorem 4.2.1. Let $X$ be a random variable with distribution function $F(x)$ and suppose that $S(x)$ is a finite and single-valued function of $x$. The characteristic function of the random variable $Y=S(X)$ is then given by

$$
\Phi_{Y}(t)=E\left(e^{i t S(X)}\right)=\int_{-\infty}^{\infty} e^{i t S(x)} d F(x) .
$$

The characteristic function of ISP circular distribution is obtained by applying the above theorem that gives the following theorem.

Theorem 4.2.2. Let $\theta$ be defined as ISP for a linear random variable $X$, then the characteristic function of ISP circular model is

$$
\phi_{\theta}(p)=\Phi_{2 \tan ^{-1}\left(\frac{X}{v}\right)}(p), \quad p \in \mathbb{Z}
$$

Proof. Let $G(\theta)$ be the distribution function of $\theta$ corresponding to ISP transformation of $X$ with the distribution function $F(x)$, then we have

$$
\begin{aligned}
\phi_{\theta}(p) & =\int_{-\pi}^{\pi} e^{i p \theta} d(G(\theta)), \quad p \in \mathbb{Z} \\
& =\int_{-\infty}^{\infty} e^{i p \theta} d\left(F\left(v \tan \left(\frac{\theta}{2}\right)\right)\right) \quad \text { by using equation }(2.4) \\
& =\int_{-\infty}^{\infty} e^{i p\left(2 \tan ^{-1}\left(\frac{x}{v}\right)\right)} d F(x) \quad \text { taking } x=v \tan \left(\frac{\theta}{2}\right) \\
& =\Phi_{2 \tan ^{-1}\left(\frac{x}{v}\right)}(p)
\end{aligned}
$$




\section{The characteristic function of the proposed ISP skewed- $t$ distribution}

$$
\phi_{\theta}(p)=\int_{-\infty}^{\infty} e^{i p \theta} g(\theta) d \theta
$$

We calculate characteristic function by using different $g(\theta)$ in equation (4.1) for three

different proposed ISP skewed- $t$ density $g_{J F}(\theta), g_{A}(\theta)$ and $g_{T P}(\theta)$. As the integral cannot be obtained analytically, R programming techniques are applied for the evaluation of the values of the characteristic function. In the next section we will discuss the skewness and kurtosis of the proposed circular distribution graphically.

\subsection{Skewness}

Moments of the new proposed distributions are not particularly tractable, however, they can be calculated numerically.

\subsubsection{Skewness of Jones and Faddy (JF) ISP skewed- $t$ distri- bution}

In Jones and Faddy (2003) skewed-t distribution (3.1), we discussed about the skewness parameters $a$ and $b$, where $a>b$ indicates positive skewness and $a<b$ indicates negative skewness. When $a=b$ then the distribution becomes symmetric. 


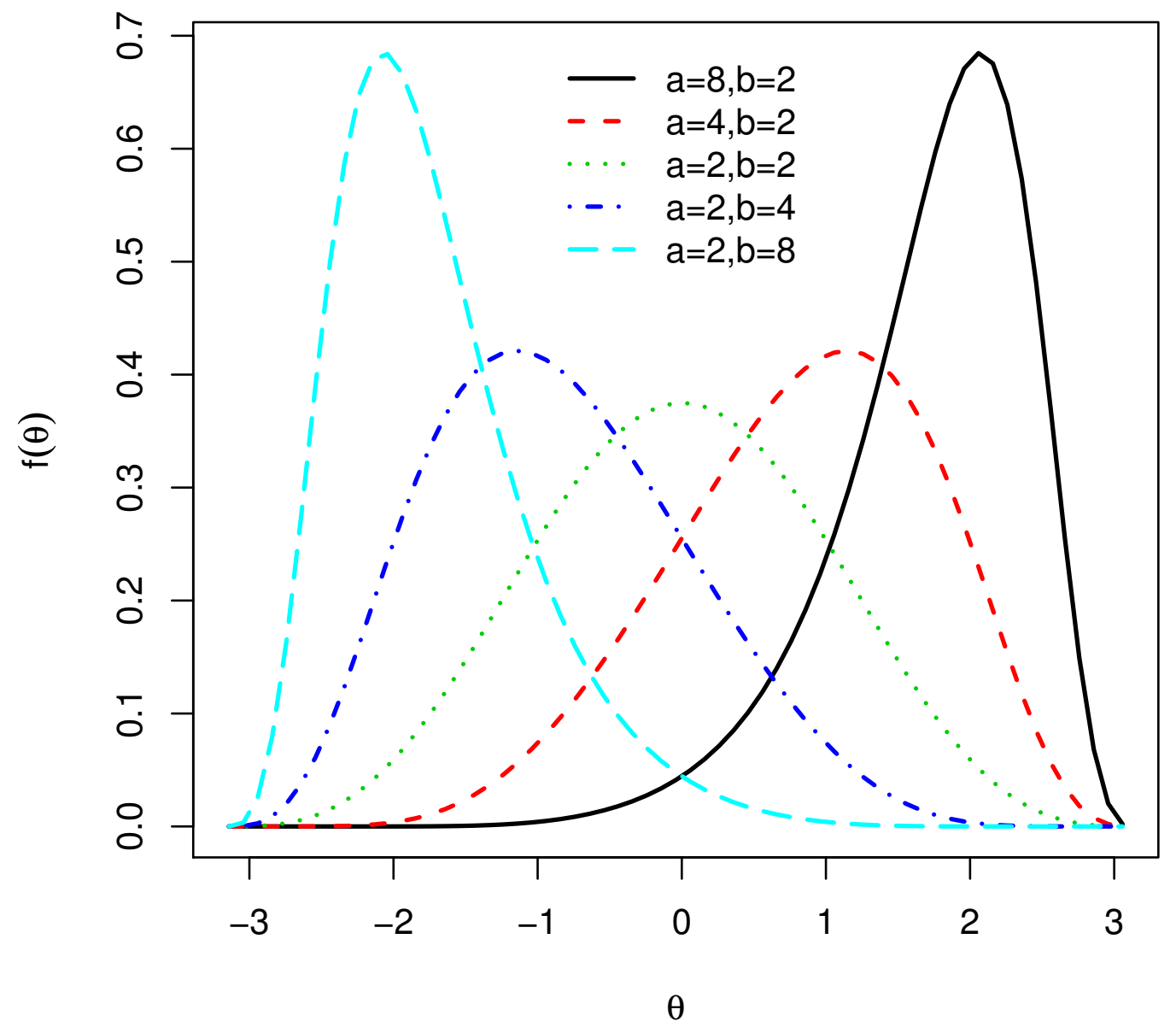

Figure 4.1: Probability density functions of Jones and Faddy ISP skewed- $t$ distribution for different values of $a$ and $b$

Similarly, as shown in Figure 4.1, the circular skewness for Jones and Faddy (JF) is zero for $a=b$, positive for $a>b$ and negative for $a<b$. The asymmetric function, and scalar measures of skewness are directly controlled by $a$ and $b$, suggesting that $a$ and $b$ itself is an excellent skewness measure for this distribution. Similar pattern is found for reparametrized JF ISP skewed-t distribution. New skewness parameter $\gamma>1$ leading to positive skewness and $\gamma<1$ corresponds to negative skewness and $\gamma=1$ gives a symmetric distribution as in Figure 4.2 


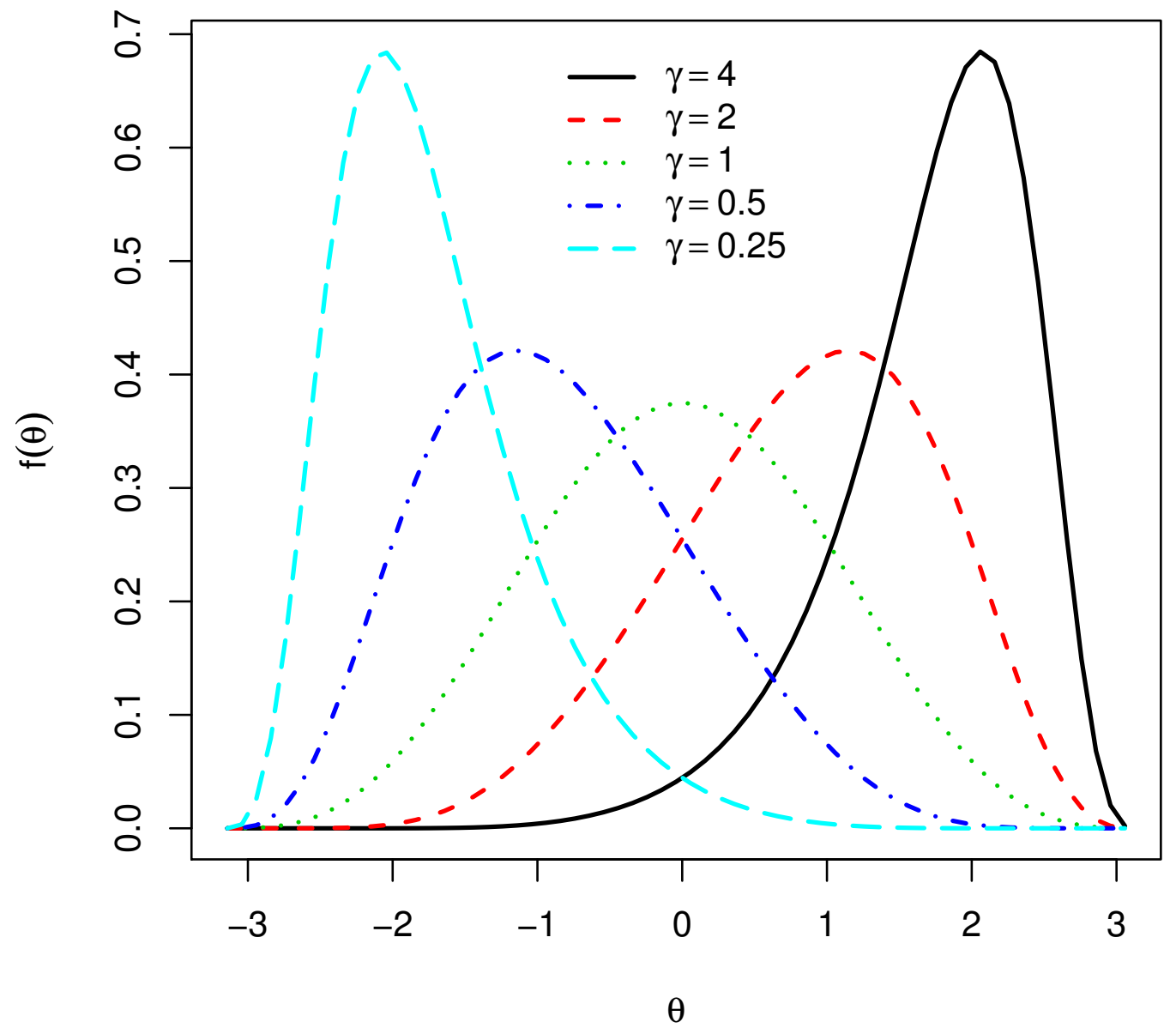

Figure 4.2: Probability density functions of reparametrized JF ISP skewed- $t$ distribution for different values of $\gamma$

\subsubsection{Skewness of Azzalini type ISP skewed- $t$ distribution}

For Azzalini type (1985) skewed-t distribution in Section 3.2.2, we discussed about the skewness parameters $\alpha$, where $\alpha>0$ indicates positive skewness and $\alpha<0$ indicates negative skewness. For $\alpha=0$ the distribution becomes a symmetric distribution. 


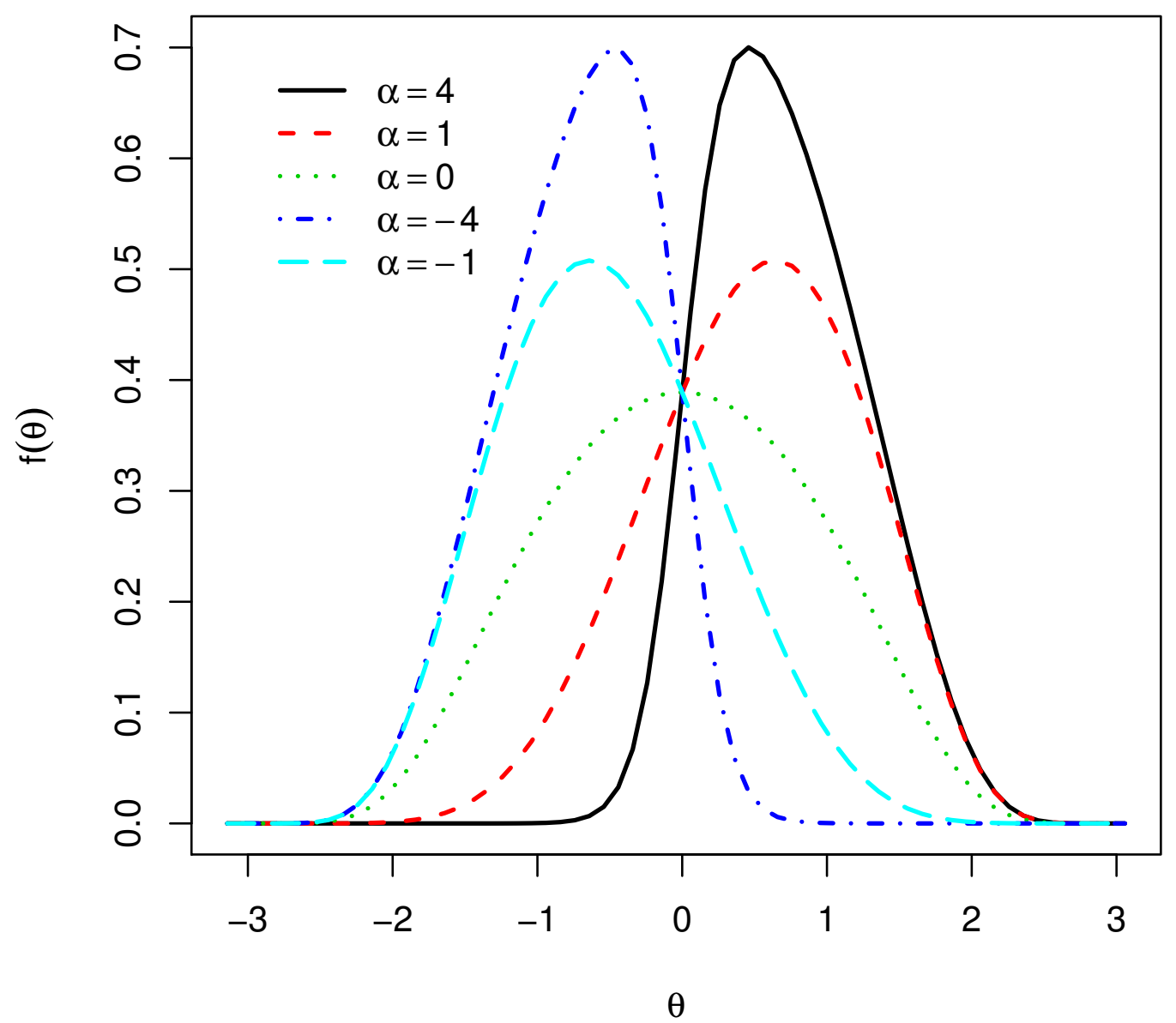

Figure 4.3: Probability density functions of Azzalini type ISP skewed- $t$ distribution for different values of $\alpha$

As shown in Figure 4.3, similar shape is found for ISP circular distribution, strongly suggesting positively skew for $\alpha>0$ and negatively skew for $\alpha<0$. Symmetric density is obtained for $\alpha=0$.

\subsubsection{Skewness of two-piece ISP skewed- $t$ distribution}

Skewness of two-piece skewed- $t$ distribution is discussed in Section 3.2.3, where $\gamma>0$ leading to negative skewness and $\gamma<0$ corresponding to positively skew and $\gamma=0$ 
tends to symmetric distribution.

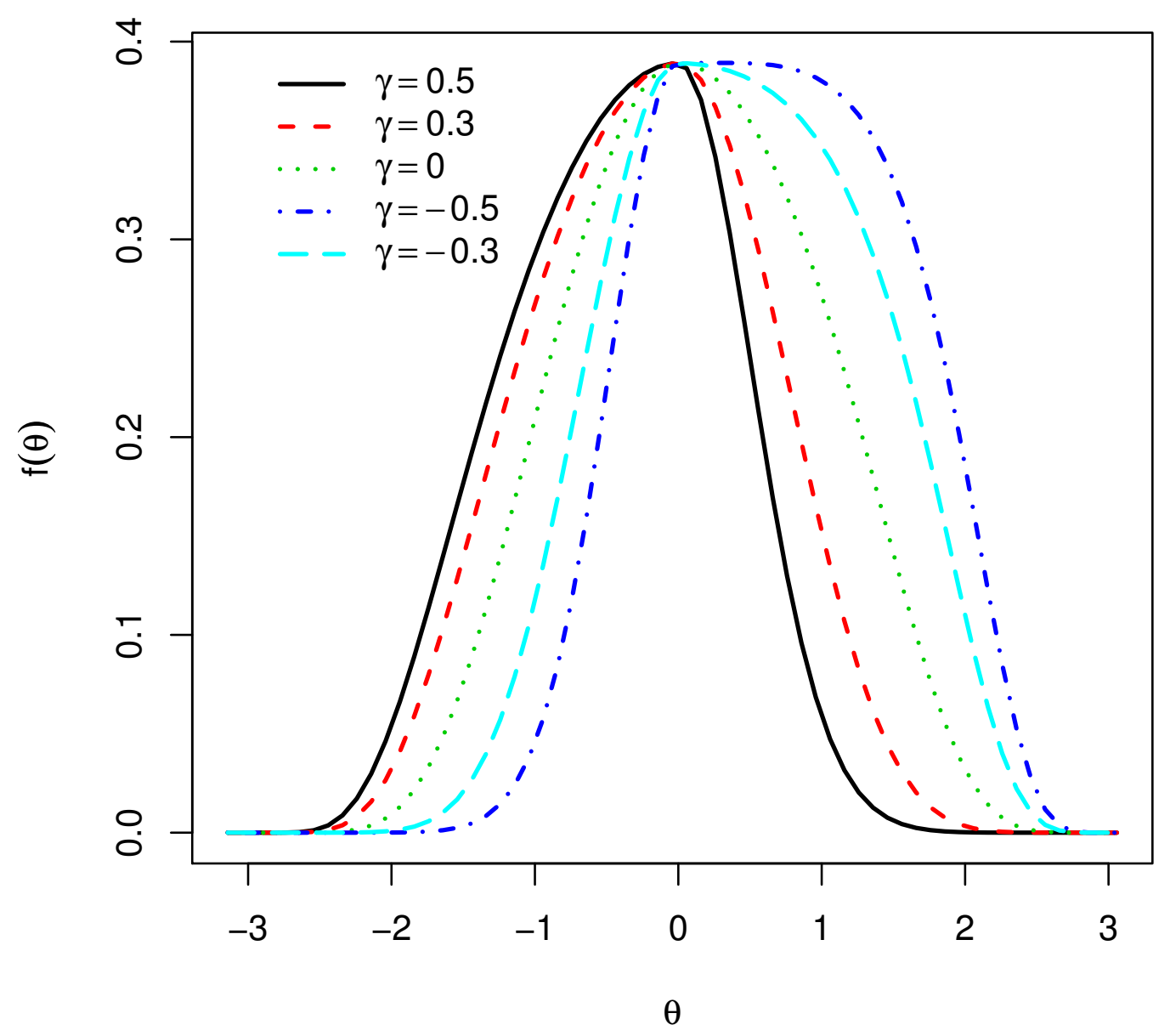

Figure 4.4: Probability density functions of two-piece ISP skewed-t distribution for different values of $\gamma$

Similarly, the circular skewness for two-piece ISP $t$-distribution is zero for $\gamma=0$, it positive for $\gamma<0$ and negative for $\gamma>0$ as presented in Figure 4.4. 


\section{Chapter 5}

\section{Estimation of Parameters in ISP Skewed- $t$ Distribution}

\section{$5.1 \quad$ Introduction}

The general theory and properties of maximum likelihood (ML) estimates have been discussed in many statistical text books. In this chapter we will discuss maximum likelihood method for the estimation of parameters of various ISP circular distributions introduced in this thesis using some real data. Likelihood based inference has the appeal that can readily be extended to address problems such as hypothesis testing, confidence set construction and finite mixture modelling.

\subsection{Maximum likelihood estimation}

We can obtain maximum likelihood parameter estimates for the distributions discussed in Section 3.3 by identifying the parameter values which maximize the likelihood function or equivalently maximize the log-likelihood function.

MLE for Jones and Faddy ISP skewed- $t$ distribution

Suppose that $\underline{\theta}=\left\{\theta_{1}, \theta_{2}, \ldots . \theta_{n}\right\}$ is an iid sample of size $n$ from the distribution (3.9), 
then the log-likelihood function is given by

$$
\log L(\gamma, m, v ; \theta)=\sum_{i=1}^{n} \log g_{J F}\left(\theta_{i}\right)
$$

Taking derivatives of (5.1) with respect of $\gamma, m$ and $v$ and equating to zero. i.e.

$$
\begin{aligned}
& \frac{\partial L}{\partial \gamma}=0 \\
& \frac{\partial L}{\partial m}=0
\end{aligned}
$$

and

$$
\frac{\partial L}{\partial v}=0
$$

Solving equations (5.2) to (5.4) simultaneously we can find the maximum likelihood estimates for the parameters. No explicit forms for the estimates are available but we can find the estimates for a particular sample of directional data by using numerical methods.

Similarly, the maximum likelihood estimates of the parameter for the Azzalini type ISP skewed- $t$ and the two-piece ISP skewed- $t$ distribution are found by using numerical optimization.

\subsection{Data Analysis}

In order to exemplify the methodology discussed in the previous sections and its extension to finite mixture modelling, we consider three different data sets that have been in the literature, the first is known as 'Bird Migration Heading Data' the second one known as the 'Drosophila Larval Locomotion Data' and the third one is 'Ants data'. (see Bruderer and Jenni (1990), Holzmann et al. (2006) and Fisher (1993) respectively). Mixture modelling is used to solve various inferential problems associated with the application of the proposed ISP circular distribution. We will fit our three 
different proposed ISP circular models to the data for visual inspection by estimating the parameters by the maximum likelihood method. We will also consider mixtures of these models with the circular uniform distribution in order to avoid zero value of the density at the tails.

Our comparison will be based on the values of Chi-square goodness-of-fit statistic and AIC that is defined as

$$
A I C=-2 \log _{e} \text { Likelihood }+2 p
$$

where $p$ is the number of parameters in the model. Even though, log-likelihood values are good enough for respective comparison of different circular distributions with same number of parameters, AIC values are useful for cross comparison among the models with different number of parameters, such as mixture and non-mixture models. Hence, we have displayed both of these in the tables. Additionally, we have also displayed the $P$ - values corresponding to the $\chi^{2}$-values that may enable us to judge the adequacy of the fitted distributions.

\subsubsection{Bird migration headings data}

The bird migration headings data set introduced to the ornithological literature by Bruderer and Jenni (1990). The data consists of the 'headings' of 1827 migrating birds recorded at an observational post near Stuttgart during the autumnal migration period of 1987. Here, the term 'heading' refers to the direction, measured in a clockwise direction from North, of a bird's body during flight.

Table 5.1: Parameters estimated for Bird migration headings data

\begin{tabular}{|l|c|}
\hline Distribution & ML Estimates \\
\hline Jones and Faddy ISP skewed- $t$ & $\widehat{m}=5.08, \widehat{\gamma}=2.11, \widehat{v}=2.62$ \\
\hline Azzalini type ISP skewed- $t$ & $\widehat{v}=1.88, \widehat{\alpha}=1.58, \widehat{\nu}=2.98$ \\
\hline Two-piece ISP skewed- $t$ & $\widehat{v}=2.08, \widehat{\gamma}=-0.52, \widehat{\nu}=3.36$ \\
\hline
\end{tabular}


Table 5.2: Comparison of fit for Bird migration headings data

\begin{tabular}{lcccc}
\hline Distribution & Max Log Likelihood & AIC & $\chi^{2}$ & $P$-value \\
\hline Jones and Faddy ISP skewed- $t$ & -2186.9 & 4379.9 & 208.30 & 0.000 \\
Azzalini type ISP skewed- $t$ & -2163.9 & 4333.9 & 154.71 & 0.000 \\
Two-piece ISP skewed- $t$ & -2354.1 & 4714.1 & 428.77 & 0.000 \\
\hline
\end{tabular}

Tables 5.1 and 5.2 shows the parameter estimates, maximized log-likelihood, AIC and chi-square values for three different distributions. The histogram of the data with estimated densities is given in Figure 5.1 for visual inspection.

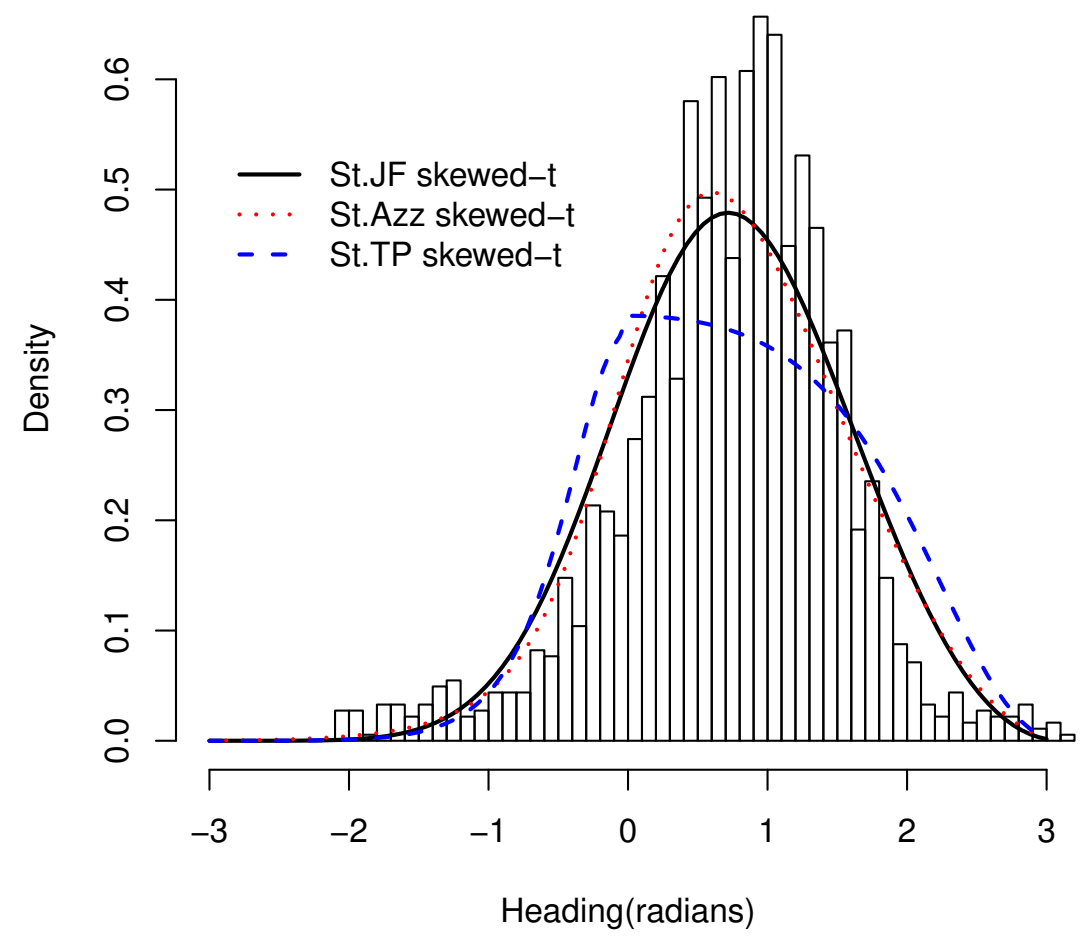

Figure 5.1: Histogram of the 1827 bird-flight headings together with fitted densities

According to Table 5.2, Azzalini type ISP skewed- $t$ model gives better fit compared to other distributions, based on the likelihood, AIC as well as chi-square values. 
However, visually, ISP Jones and Faddy skewed- $t$ and Azzalini type skewed- $t$ is very similar and it is clear that neither provides good fit to the data. Specifically, neither is capable of simultaneously modelling the peakedness and long 'tails' of the histogram. Moreover, zero density at the tails that is necessitated by all the new distributions creates a problem for modelling.

\subsubsection{Mixture Distributions}

To overcome the problem of modelling the peakedness and long 'tails' simultaneously, we consider an empirical model by mixing the circular uniform distribution with the proposed ISP skewed- $t$ distribution (Pewsey, 2006). The density of the empirical model which corresponds to mixture distribution with circular uniform and ISP skewed- $t$ components.

$$
\begin{gathered}
g_{M J F}(\theta ; m, v, \gamma, w)=\frac{w}{2 \pi}+(1-w) * g_{J F}(\theta ; m, v, \gamma) \\
g_{M A}(\theta ; \nu, v, \alpha, w)=\frac{w}{2 \pi}+(1-w) * g_{A}(\theta ; \nu, v, \alpha) \\
g_{M T P}(\theta ; \nu, v, \gamma, w)=\frac{w}{2 \pi}+(1-w) * g_{T P}(\theta ; \nu, v, \gamma)
\end{gathered}
$$

where $g_{M J F}(\theta ; m, v, \gamma, w), g_{M A}(\theta ; \nu, v, \alpha, w)$ and $g_{M T P}(\theta ; \nu, v, \gamma, w)$ are the mixture ISP Jones and Faddy, Azzalini type and two-piece skewed- $t$ distribution respectively and $\mathrm{w}$ being the mixing probability associated with the two components. The ML solution obtained by using log-likelihood derived from equation (5.6) to (5.8) and their comparison of fit is presented in Tables 5.3 and 5.4.

Table 5.3: Parameters estimated for mixture distribution for Bird migration headings data

\begin{tabular}{|l|c|}
\hline Distribution & ML Estimates \\
\hline Jones and Faddy ISP skewed- $t$ & $\widehat{m}=17.38, \widehat{\gamma}=1.75, \widehat{v}=2.86, \widehat{w}=0.096$ \\
\hline Azzalini type ISP skewed- $t$ & $\widehat{v}=1.65, \widehat{\alpha}=2.29, \widehat{\nu}=801.4, \widehat{w}=0.103$ \\
\hline Two-piece ISP skewed- $t$ & $\widehat{v}=2.34, \widehat{\gamma}=-0.67, \widehat{\nu}=1599.9, \widehat{w}=0.117$ \\
\hline
\end{tabular}


Table 5.4: Comparison of fit for mixture distribution for Bird migration headings data

\begin{tabular}{lcccc}
\hline Distribution & Max Log Likelihood & AIC & $\chi^{2}$-value & $P$-value \\
\hline Jones and Faddy ISP skewed- $t$ & -2098.1 & 4204.3 & 28.57 & 0.195 \\
Azzalini type ISP skewed- $t$ & -2111.6 & 4231.2 & 37.62 & 0.028 \\
Two-piece ISP skewed- $t$ & -2225.0 & 4458.1 & 193.35 & 0.000 \\
\hline
\end{tabular}

Table 5.4 shows that the mixture of the circular uniform distribution with Jones and Faddy ISP skewed- $t$ gives a better fit compared to others distributions, according to the likelihood, AIC as well as chi-square values where as the two-piece ISP skewed- $t$ provides a very poor fit. The mixture modeling improves both Jones and Faddy ISP skewed- $t$ and Azzalini type ISP skewed- $t$ distributions as plausible models, however, only Jones and Faddy type ISP skewed $-t$ seems meaningful according to the $P$-value. Note that two-piece ISP skewed- $t$ does not provide a good fit even after mixing with circular uniform. 


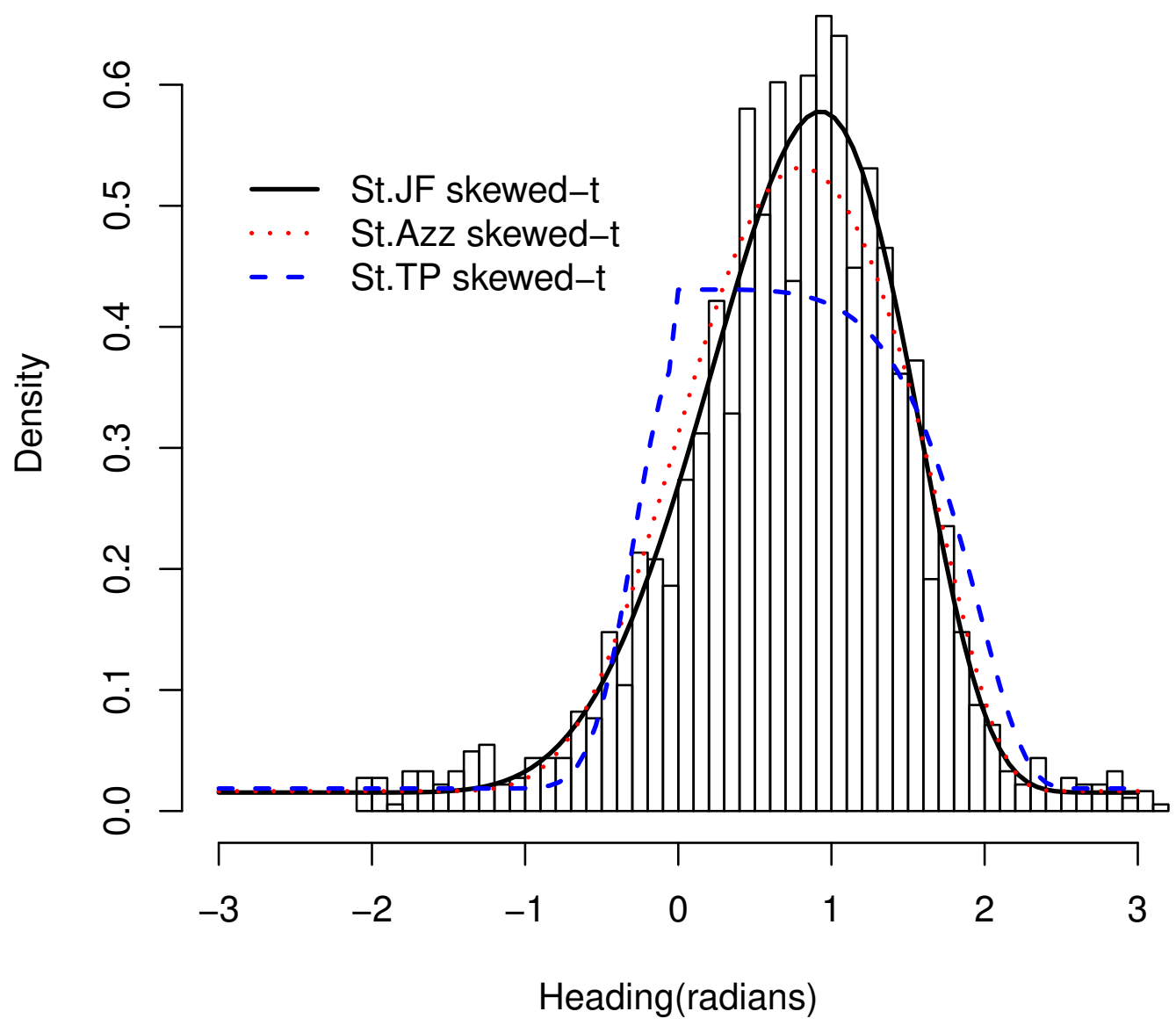

Figure 5.2: Histogram of the 1827 bird-flight headings together with fitted mixture densities

\subsubsection{Drosophila larval locomotion data}

In our second example, we present an analysis of the $n=180$ changes in direction of a single Drosophila fly larva, measured once per second over a period of three minutes (Holzmann et al., 2006). The maximum likelihood estimates of the parameters including maximized log-likelihood, AIC and chi-square values for three proposed ISP circular distribution are presented in Tables 5.5 and 5.6. A histogram of the data 
with fitted densities is portrayed in Figure 5.3.

Table 5.5: Parameters estimated for Drosophila larval locomotion data

\begin{tabular}{|l|c|}
\hline Distribution & ML Estimates \\
\hline Jones and Faddy ISP skewed- $t$ & $\widehat{m}=1.11, \widehat{\gamma}=0.79, \widehat{v}=10.64$ \\
\hline Azzalini type ISP skewed- $t$ & $\widehat{v}=10.36, \widehat{\alpha}=-0.227, \widehat{\nu}=1.08$ \\
\hline Two-piece ISP skewed- $t$ & $\widehat{v}=2.61, \widehat{\gamma}=0.201, \widehat{\nu}=2.57$ \\
\hline
\end{tabular}

Table 5.6: Comparison of fit for Drosophila larval locomotion data

\begin{tabular}{lcccc}
\hline Distribution & Max Log Likelihood & AIC & $\chi^{2}$-value & $P$-value \\
\hline Jones and Faddy ISP skewed- $t$ & -113.98 & 233.96 & 1.29 & 0.731 \\
Azzalini type ISP skewed- $t$ & -114.86 & 235.71 & 1.31 & 0.726 \\
Two-piece ISP skewed- $t$ & -228.64 & 463.27 & 19.10 & 0.000 \\
\hline
\end{tabular}

Table 5.6 illustrates that Jones and Faddy ISP skewed- $t$ model provides better fit compared to other distributions, according to the likelihood, AIC as well as chi-square values. The visual inspection of Figure 5.3 shows that both Jones and Faddy type ISP skewed- $t$ and Azzalini type ISP skewed- $t$ provide similar fits (actually they overlap each other), where as the two-piece ISP skewed- $t$ provides a very poor fit. This is also confirmed using the AIC and $\chi^{2}-$ values. Both Jones and Faddy skewed- $t$ and Azzalini type ISP skewed- $t$ distributions are accepted as good models where as the two-piece ISP skewed- $t$ is rejected. 


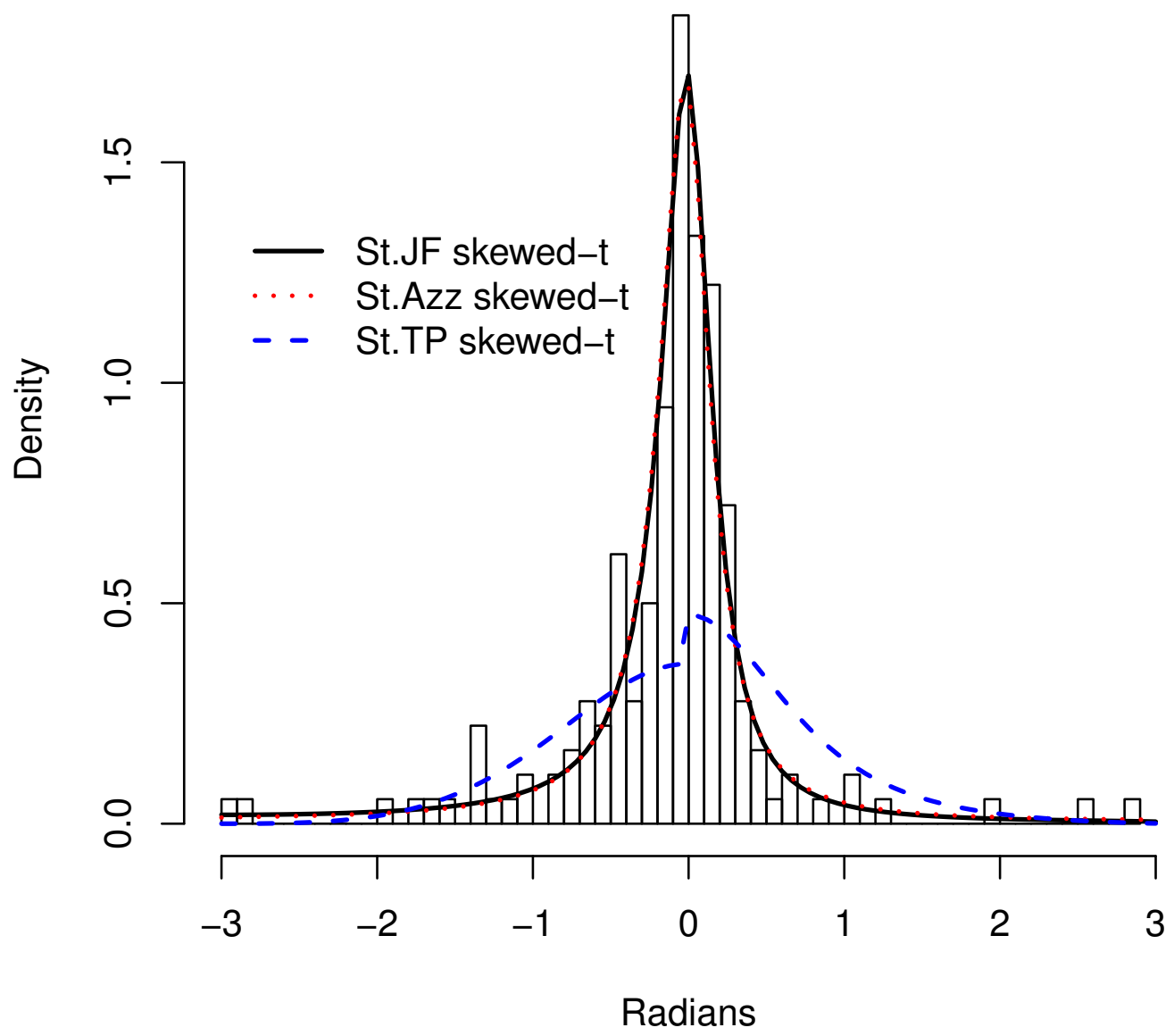

Figure 5.3: Histogram of the Drosophila larval locomotion data together with fitted densities

Table 5.7: Parameters estimated for mixture distribution for Drosophila larval locomotion data

\begin{tabular}{|l|c|}
\hline Distribution & ML Estimates \\
\hline Jones and Faddy ISP skewed- $t$ & $\widehat{m}=1.27, \widehat{\gamma}=0.77, \widehat{v}=10.51, \widehat{w}=0.0266$ \\
\hline Azzalini type ISP skewed- $t$ & $\widehat{v}=10.31, \widehat{\alpha}=-0.212, \widehat{\nu}=0.911, \widehat{w}=0.06$ \\
\hline Two-piece ISP skewed- $t$ & $\widehat{v}=2.79, \widehat{\gamma}=0.2307, \widehat{\nu}=1215, \widehat{w}=0.0727$ \\
\hline
\end{tabular}


Table 5.8: Comparison of fit for mixture distribution for Drosophila larval locomotion

\begin{tabular}{lcccc} 
data & Max Log Likelihood & AIC & $\chi^{2}$-value & $P$-value \\
\hline Distribution & -113.85 & 235.70 & 1.02 & 0.797 \\
Jones and Faddy ISP skewed- $t$ & -114.64 & 237.27 & 4.17 & 0.360 \\
Azzalini type ISP skewed- $t$ & -207.21 & 422.41 & 18.34 & 0.000 \\
Two-piece ISP skewed- $t$ & &
\end{tabular}

Tables 5.7 and 5.8 present analogous results to those presented in Tables 5.5 and 5.6. Moreover, Figure 5.4 looks similar to Figure 5.3. Becaue of the ISP Jones and Faddy skewed- $t$ and Azzalini type skewed- $t$ provides good fit except two-piece, mixing ISP skewed- $t$ with circular uniform do not have significant effect for this data. 


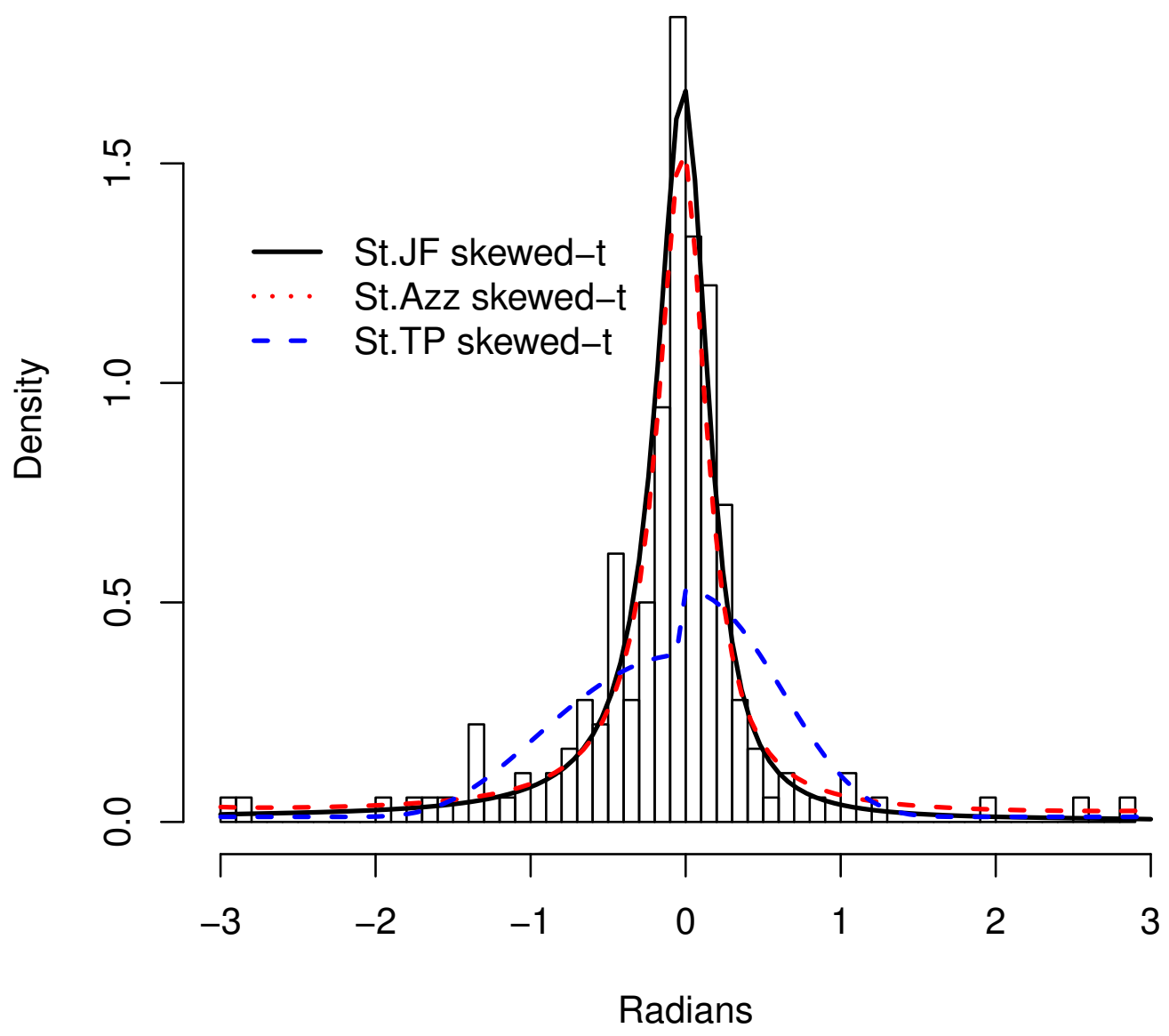

Figure 5.4: Histogram of the Drosophila larval locomotion data together with fitted mixture densities

\subsubsection{Ants data}

In our third example we consider the ants data which consists of directions chosen by 100 ants in response to an evenly illuminated black target as described in Fisher (1993). Tables 5.9 and 5.10 illustrates the parameter estimates, maximized log-likelihood, AIC and chi-square values for three different proposed ISP circular distributions. We also plotted the histogram of the data with fitted densities in Fig- 
ure 5.5. According to Table 5.10, comparing AIC and chi-square values, as well as looking at the Figure 5.5, we find that ISP Jones and Faddy skewed- $t$ and Azzalini type skewed- $t$ fits are very close where as the two-piece ISP skewed- $t$ has a very poor fit. These conclusions are also confirmed according to the $P$ - values. It is well known from Jones and Faddy (2003), that the parameter $\gamma$ for the two-piece skewed $-t$ distribution for the real line can not accommodate both the peakedness and long tails; this may explain the poor fit here. We also fitted the mixture model (results not given here) but did not see any improvement in the fit.

Table 5.9: Parameters estimated for Ants data

\begin{tabular}{|l|c|}
\hline Distribution & ML Estimates \\
\hline Jones and Faddy ISP skewed- $t$ & $\widehat{m}=0.87, \widehat{\gamma}=1.074, \widehat{v}=4.94$ \\
\hline Azzalini type ISP skewed- $t$ & $\widehat{v}=4.97, \widehat{\alpha}=0.142, \widehat{\nu}=0.86$ \\
\hline Two-piece ISP skewed- $t$ & $\widehat{v}=1.91, \widehat{\gamma}=0.00029, \widehat{\nu}=1.324$ \\
\hline
\end{tabular}

Table 5.10: Comparison of fit for Ants data

\begin{tabular}{lcccc}
\hline Distribution & Max Log Likelihood & AIC & $\chi^{2}$-value & $P$-value \\
\hline Jones and Faddy ISP skewed- $t$ & -132.28 & 270.56 & 3.55 & 0.314 \\
Azzalini type ISP skewed- $t$ & -131.94 & 269.89 & 3.21 & 0.359 \\
Two-piece ISP skewed- $t$ & -170.05 & 346.09 & 39.16 & 0.000 \\
\hline
\end{tabular}




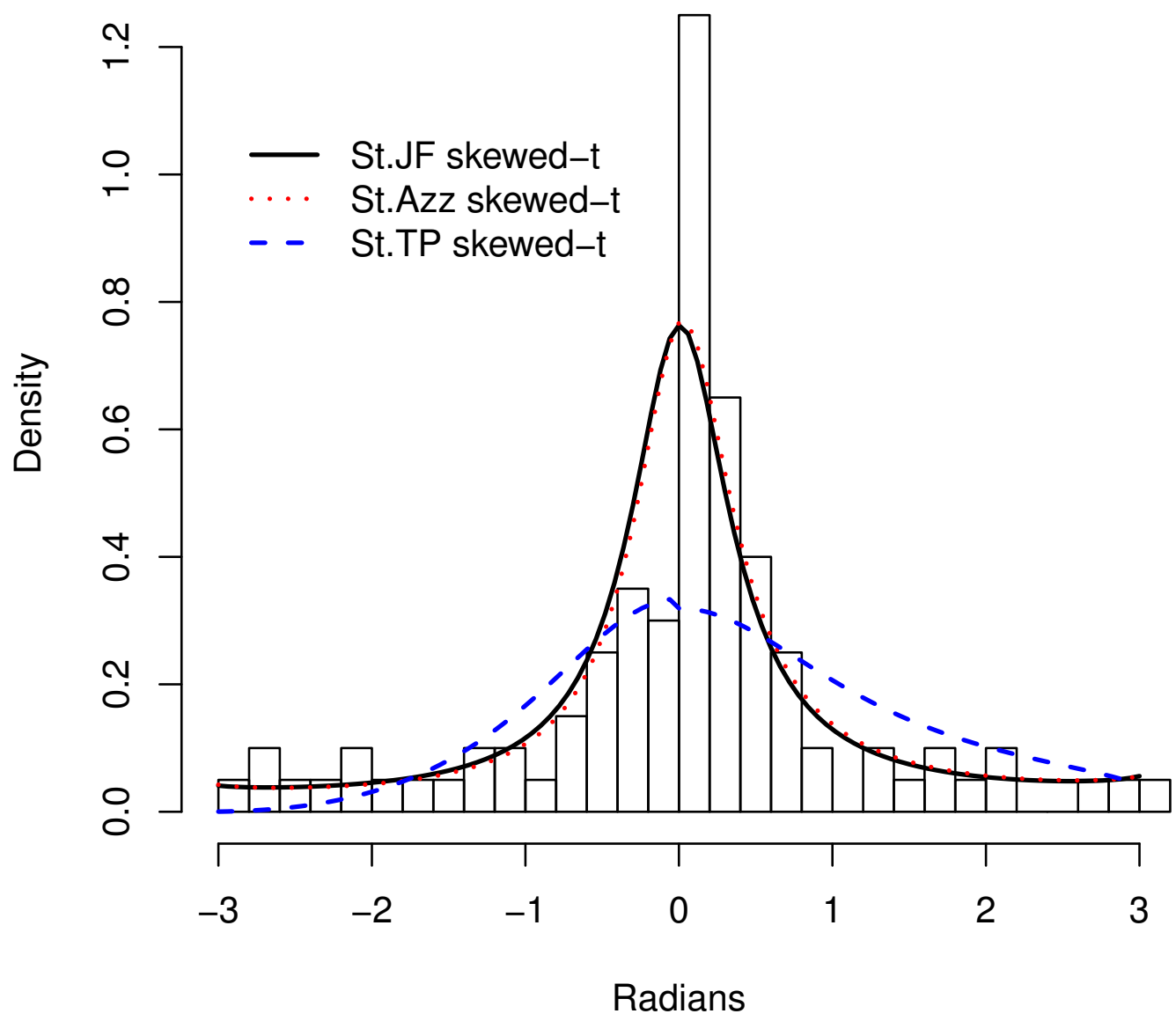

Figure 5.5: Histogram of the Ants data together with fitted densities 


\section{Chapter 6}

\section{Conclusions}

The basic objective of this thesis is to provide flexible and numerically tractable probability models for circular data. In this regard we find that inverse stereographic projection (ISP) method of transforming a distribution on the real line to that on the perimeter of a unit circle. There have been many families of asymmetric distributions proposed earlier in the literature which offer a comprehensive varieties of forms such as that due to Kato and Jones (2010). However, such families involve intensive computations where as for the new families of distributions based on various skewed$t$ families, explicit form of the density function is available.

In order to judge the flexibility of shapes, skewness of each of the resulting distribution is investigated. On this account we have some success, however, it is not able to incorporate heavy tails as the density at the tails is necessarily zero. In order to alleviate this problem a mixture model that mixes the resulting ISP circular distribution with the circular uniform distribution. The skewed- $t$ families chosen for investigation are those due to Faddy and Jones (2003), Azzalini (1985) and two-piece skewed- $t$ considered by Rosco et al. (2011).

We have also studied three practical examples with respect to the suitability of the new models.. We compared the rankings for the models fitted to three different practical data based on different criteria (maximized log-likelihood, AIC and chi-square 
values), and showed that they can provide somewhat different rankings. Example 1 (Bird migration headings data) illustrates that the Jones and Faddy ISP skewed- $t$ distribution provides a flexible model for asymmetrically distributed circular data, particularly when used as a component in finite mixture modelling. On the other hand, two-piece ISP skewed-t distribution does not fit well. Example 2 (Drosophila Larval Locomotion data) shows that ISP Jones and Faddy skewed- $t$ and Azzalini type skewed- $t$ fit very well considering peakedness and long 'tails' of the histogram without finite mixture modelling. The fitted densities are overlapped for ISP Jones and Faddy skewed- $t$ and Azzalini type skewed- $t$. Similar results are also obtained for Example 3 (Ants data). That is the estimated densities of ISP Jones and Faddy skewed- $t$ and Azzalini type skewed- $t$ are overlapping each other. However, ISP circular distribution obtained from two-piece skewed- $t$ does not fit good for any of three examples. The graph of this distribution is not of very appealing nature as the two pieces seem to be fitted forcibly.

The incorporation of a mixture with the uniform circular distribution basically lifts the tails and provides a very flexible model as seen from the examples. The ISP circular distribution obtained from the Azzalini type skewed- $t$ involves the distribution function of the Student's $t$ distribution, hence is not computationally attractive over that obtained by Jones and Faddy skewed- $t$. Hence we can conclude that the ISP circular distribution obtained from Jones and Faddy skewed- $t$ is an attractive alternative to other asymmetric unimodal circular distributions, especially when combined with a mixture of uniform circular distribution.

In this thesis, we have emphasized on generating new skewed circular distributions, but have not paid much attention to testing or confidence intervals. This may be pursued in further research. 


\section{Bibliography}

Abe, T. and Pewsey, A. (2011). Sine-skewed circular distributions. Statistical Papers, $52(3): 683-707$.

Abe, T., Pewsey, A., and Shimizu, K. (2009). On papakonstantinou's extension of the cardioid distribution. Statistics \& Probability Letters, 79(20):2138-2147.

Abe, T., Shimizu, K., and Pewsey, A. (2010). Symmetric unimodal models for directional data motivated by inverse stereographic projection. Journal of the Japan Statistical Society, 40(1):045-061.

Arnold, B. C. and SenGupta, A. (2006). Probability distributions and statistical inference for axial data. Environmental and Ecological Statistics, 13(3):271-285.

Azzalini, A. (1985). A class of distributions which includes the normal ones. Scandinavian journal of statistics, pages 171-178.

Azzalini, A. and Capitanio, A. (1999). Statistical applications of the multivariate skew normal distribution. Journal of the Royal Statistical Society: Series B (Statistical Methodology), 61(3):579-602.

Azzalini, A. and Capitanio, A. (2003). Distributions generated by perturbation of symmetry with emphasis on a multivariate skew t-distribution. Journal of the Royal Statistical Society: Series B (Statistical Methodology), 65(2):367-389.

Batschelet, E. (1981). Circular statistics in biology. London : Academic. 
Beran, R. (1979). Exponential models for directional data. The Annals of Statistics, pages $1162-1178$.

Branco, M. D. and Dey, D. K. (2001). A general class of multivariate skew-elliptical distributions. Journal of Multivariate Analysis, 79(1):99-113.

Bruderer, B. and Jenni, L. (1990). Migration across the alps. In Bird migration, pages 60-77. Springer.

Chaubey, Y. and Midhu, N. (2015). Circular distribution arising from the möbius transformation of wrapped distributions. Proceedings of IMBIC, The 9th International Conference of IMBIC, MSAST,Kolkata,India, 2015(4):6-24.

Chaubey, Y. P. (2016). Smooth kernel estimation of a circular density function: A connection to orthogonal polynomials on the unit circle. arXiv preprint arXiv:1601.05053.

Dattatreya Rao, A., Girija, S., and Devaraaj, V. (2013). On the rising sun wrapped lognormal and the rising sun wrapped exponential models. International Journal of Statistics and Systems, 3(1):1-10.

Dattatreya Rao, A., Girija, S., and Phani, Y. (2011). On stereographic logistic model. Proceedings of NCAMES, AU Engineering College, Visakhapatnam, pages 139-141.

Dattatreya Rao, A., Ramabhadra Sarma, I., and Girija, S. (2007). On wrapped version of some life testing models. Communications in Statistics-Theory and Methods, 36(11):2027-2035.

Di Marzio, M., Panzera, A., and Taylor, C. C. (2009). Local polynomial regression for circular predictors. Statistics \& Probability Letters, 79(19):2066-2075.

Fernández, C. and Steel, M. F. (1998). On bayesian modeling of fat tails and skewness. Journal of the American Statistical Association, 93(441):359-371. 
Fernández-Durán, J. (2004). Circular distributions based on nonnegative trigonometric sums. Biometrics, 60(2):499-503.

Fernández-Durán, J. (2007). Models for circular-linear and circular-circular data constructed from circular distributions based on nonnegative trigonometric sums. Biometrics, 63(2):579-585.

Ferreira, J. T. and Steel, M. F. (2007). A new class of skewed multivariate distributions with applications to regression analysis. Statistica Sinica, pages 505-529.

Fieller, N., Flenley, E., and Olbricht, W. (1992). Statistics of particle size data. Applied Statistics, pages 127-146.

Fisher, N. (1989). Smoothing a sample of circular data. Journal of structural geology, $11(6): 775-778$.

Fisher, N. (1993). Statistical analysis of circula data. Cambridge University Press.

Genton, M. G. (2004). Skew-elliptical distributions and their applications: a journey beyond normality. CRC Press.

Girija, S., Radhika, A., and Rao, A. (2013a). On bimodal offset cauchy distribution. Journal of Applied Mathematics, Statistics and Informatics, 9(1):61-67.

Girija, S., Rao, A., and Yedlapalli, P. (2014). New circular model induced by inverse stereographic projection on double exponential model-application to birds migration data. Journal of Applied Mathematics, Statistics and Informatics, 10(1):5-17.

Girija, S., Rao, A. D., and Yedlapalli, P. (2013b). On stereographic lognormal distribution. International Journal of Advances in Applied Sciences, 2(3):125-132.

Holzmann, H., Munk, A., Suster, M., and Zucchini, W. (2006). Hidden markov models for circular and linear-circular time series. Environmental and Ecological Statistics, 13(3):325-347. 
Jammalamadaka, S. R. and Kozubowski, T. (2003). A new family of circular models: The wrapped laplace distributions. Advances and applications in statistics, 3(1):77103.

Jammalamadaka, S. R. and Kozubowski, T. J. (2001). A wrapped exponential circular model. Proc. Andhra Pradesh Acad. Sci., 5(1):43-56.

Jammalamadaka, S. R. and Kozubowski, T. J. (2004). New families of wrapped distributions for modeling skew circular data. Communications in Statistics-Theory and Methods, 33(9):2059-2074.

Jammalamadaka, S. R. and SenGupta, A. (1998). Predictive inference for directional data. Statistics 8 probability letters, 40(3):247-257.

Jammalamadaka, S. R. and SenGupta, A. (2001). Topics in Circular Statistics. World Scientific Publishing Pte. Ltd, Singapore.

Johnson, N. L., Kotz, S., and Balakrishnan, N. (1995). Continuous Univariate Distributions, Volume 2, 2nd Edition. Wiley.

Jones, M. (2001). A skew $t$ distribution. In Probability and Statistical Models with Applications (eds C.A.Charalambides, M.V.Koutras, N.Balakrishnan). London : Chapman and Hall.

Jones, M. (2006). A note on rescalings, reparametrizations and classes of distributions. Journal of Statistical Planning and Inference, 136(10):3730-3733.

Jones, M. (2008). The t family and their close and distant relations. Journal of the Korean Statistical Society, 37(4):293-302.

Jones, M. and Faddy, M. (2003). A skew extension of the t-distribution, with applications. Journal of the Royal Statistical Society: Series B (Statistical Methodology), $65(1): 159-174$. 
Jones, M. and Pewsey, A. (2005). A family of symmetric distributions on the circle. Journal of the American Statistical Association, 100(472):1422-1428.

Jones, M. and Pewsey, A. (2012). Inverse batschelet distributions for circular data. Biometrics, 68(1):183-193.

Julia, O. and Vives-Rego, J. (2005). Skew-laplace distribution in gram-negative bacterial axenic cultures: new insights into intrinsic cellular heterogeneity. Microbiology, 151(3):749-755.

Kato, S. and Jones, M. (2010). A family of distributions on the circle with links to, and applications arising from, möbius transformation. Journal of the American Statistical Association, 105(489):249-262.

Kato, S. and Jones, M. (2015). A tractable and interpretable four-parameter family of unimodal distributions on the circle. Biometrika, 102(1):181-190.

Kato, S., Jones, M., et al. (2013). An extended family of circular distributions related to wrapped cauchy distributions via brownian motion. Bernoulli, 19(1):154-171.

Kato, S. and Pewsey, A. (2015). A möbius transformation-induced distribution on the torus. Biometrika, page asv003.

Kent, J., Mardia, K., and Rao, J. (1979). A characterization of the uniform distribution on the circle. The Annals of Statistics, pages 882-889.

Lukacs, E. (1970). Characteristic Functions. New York: Hafner Pub.co.

Ma, Y. and Genton, M. G. (2004). Flexible class of skew-symmetric distributions. Scandinavian Journal of Statistics, 31(3):459-468.

Mardia, K. and Sutton, T. (1975). On the modes of a mixture of two von mises distributions. Biometrika, pages 699-701. 
Mardia, K. V. (1975). Statistics of directional data:journal of the royal statistical society. Journal of the Royal Statistical Society. Series B (Methodological), pages 349-393.

Mardia, K. V. and Jupp, P. E. (2009). Directional statistics.

Minh, D. L. and Farnum, N. R. (2003). Using bilinear transformations to induce probability distributions. Communications in Statistics-Theory and Methods, 32(1):1-9.

Pewsey, A. (2000). The wrapped skew-normal distribution on the circle. Communications in Statistics-Theory and Methods, 29(11):2459-2472.

Pewsey, A. (2002). Testing circular symmetry. Canadian Journal of Statistics, 30(4):591-600.

Pewsey, A. (2006). Modelling asymmetrically distributed circular data using the wrapped skew-normal distribution. Environmental and Ecological Statistics, $13(3): 257-269$.

Pewsey, A. (2008). The wrapped stable family of distributions as a flexible model for circular data. Computational Statistics $\&$ Data Analysis, 52(3):1516-1523.

Pewsey, A., Lewis, T., and Jones, M. (2007). The wrapped t family of circular distributions. Australian $\&$ New Zealand Journal of Statistics, 49(1):79-91.

Rachev, S., Menn, C., and Fabozzi, F. J. (2005). Fat-tailed and skewed asset return distributions:implications for risk management,portfolio selection, and option pricing. Wiley, New York.

Radhika, A., Girija, S., and Rao, A. (2013). On rising sun von mises and rising sun wrapped cauchy circular models. Journal of Applied Mathematics, Statistics and Informatics, 9(2):41-55. 
Rayleigh, L. (1880). On the resultant of a large number of vibrations of the same pitch and of arbitrary phase. The London, Edinburgh, and Dublin Philosophical Magazine and Journal of Science, 10(60):73-78.

Rivest, L.-P. (1982). Some statistical methods for bivariate circular data. Journal of the Royal Statistical Society. Series B (Methodological), pages 81-90.

Rosco, J., Jones, M., and Pewsey, A. (2011). Skew t distributions via the sinh-arcsinh transformation. Test, 20(3):630-652.

Sahu, S. K., Dey, D. K., and Branco, M. D. (2003). A new class of multivariate skew distributions with applications to bayesian regression models. Canadian Journal of Statistics, 31(2):129-150.

Sarma, R., Dattatreya Rao, A., and Girija, S. (2011). On characteristic functions of the wrapped lognormal and the wrapped weibull distributions. Journal of Statistical Computation and Simulation, 81(5):579-589.

Sarma, R. I., Dattatreya Rao, A., and Girija, S. (2009). On characteristic functions of wrapped half logistic and binormal distributions. International Journal of Statistics and Systems, 4(1):33-45.

SenGupta, A. and Ugwuowo, F. I. (2006). Asymmetric circular-linear multivariate regression models with applications to environmental data. Environmental and Ecological Statistics, 13(3):299-309.

Stephens, M. (1963). Random walk on a circle. Biometrika, 50(3/4):385-390.

Stephens, M. A. (1962). Exact and approximate tests for directions. i. Biometrika, $49(3 / 4): 463-477$.

Taylor, C. C. (2008). Automatic bandwidth selection for circular density estimation. Computational Statistics \& Data Analysis, 52(7):3493-3500. 
Wang, M.-z. and Shimizu, K. (2012). On applying möbius transformation to cardioid random variables. Statistical Methodology, 9(6):604-614.

Yedlapalli, P., Radhika, A., Girija, S., and Rao, A. D. (2014). New circular models induced by modified inverse stereographic projection on arc tan exponential-type distribution. International Journal of Mathematical Archive (IJMA) ISSN 2229$5046,5(4)$. 


\section{Appendix A}

\section{R-Code}

\section{A.1 R-code for raw circular data plots}

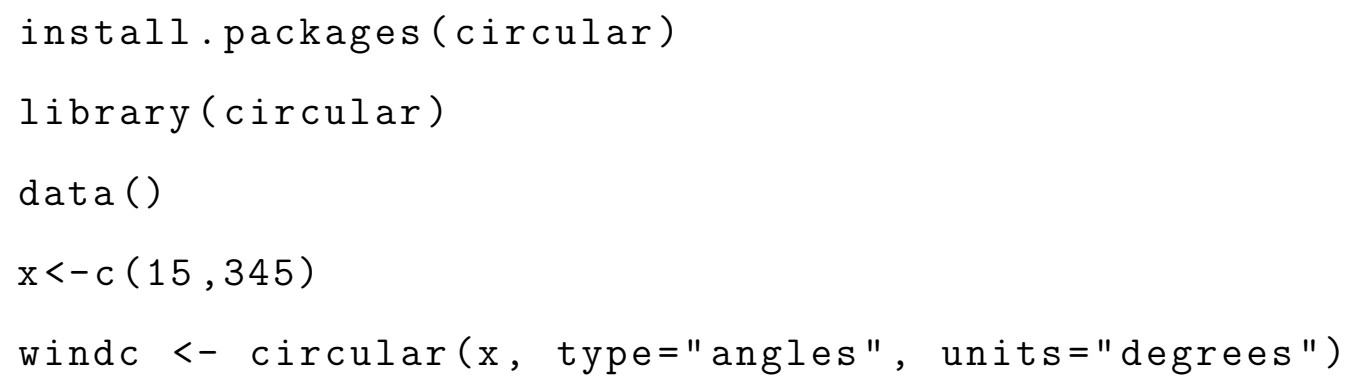

plot $($ windc, cex=1.5, bin=200, stack=TRUE, sep=0.035, shrink=1.3,

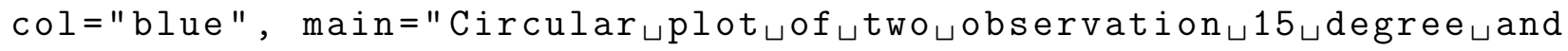
$385 \sqcup$ degree") arrows. circular (c(windc,pi), lwd=2)

\#\#\#Ant data circular plot\#\#\#\#\#\# $\operatorname{data}(f$ isherB $\mathrm{c}$ ) plot (fisherB7c, zero=pi/2, rotation='clock', col="blue", stack=TRUE, bins $=720, \operatorname{cex}=1.1, \operatorname{sep}=0.1, \operatorname{shrink}=1.5, \operatorname{xim}=c(-1,1), \operatorname{yl}$ im=c $(-2,1))$ 


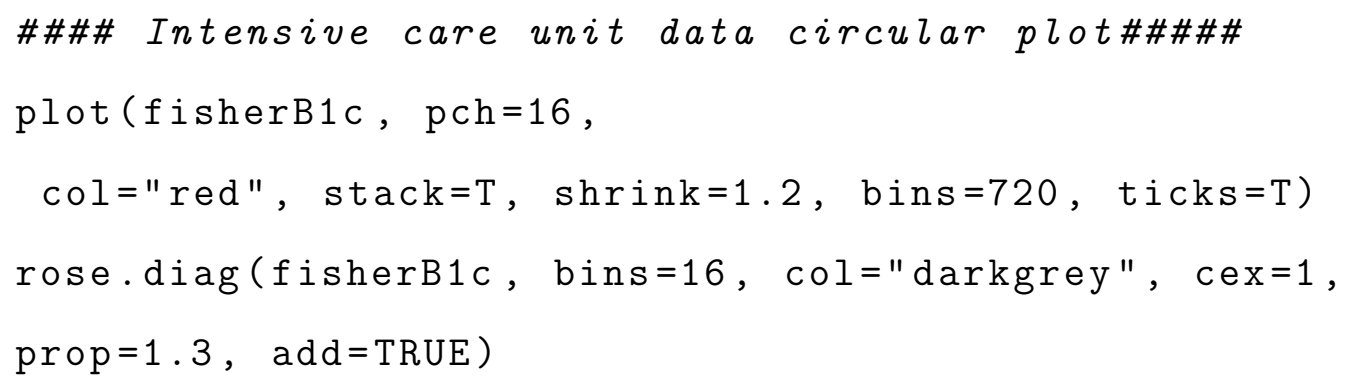

\#\#\# Histogram of bird migration headings\#\#\#\#

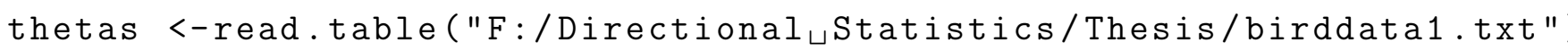
birds $<-$ thetas $\$ \mathrm{~V} 1$

hist (birds, breaks=20, $x l a b=$ "Heading (radians)", ylab="Frequency")

\section{A.2 R-code for density plot of ISP skewed- $t$ dis- tributions}

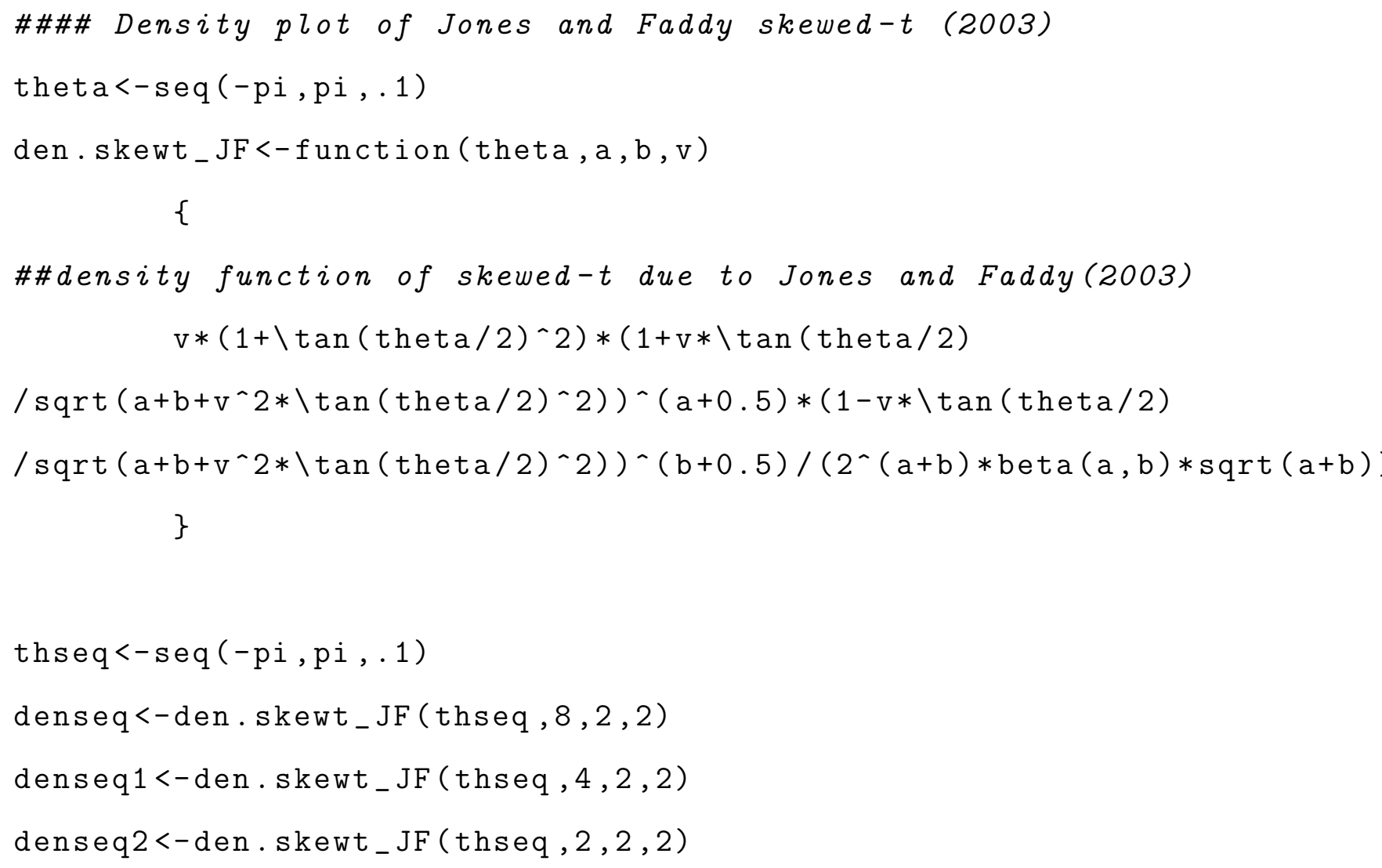




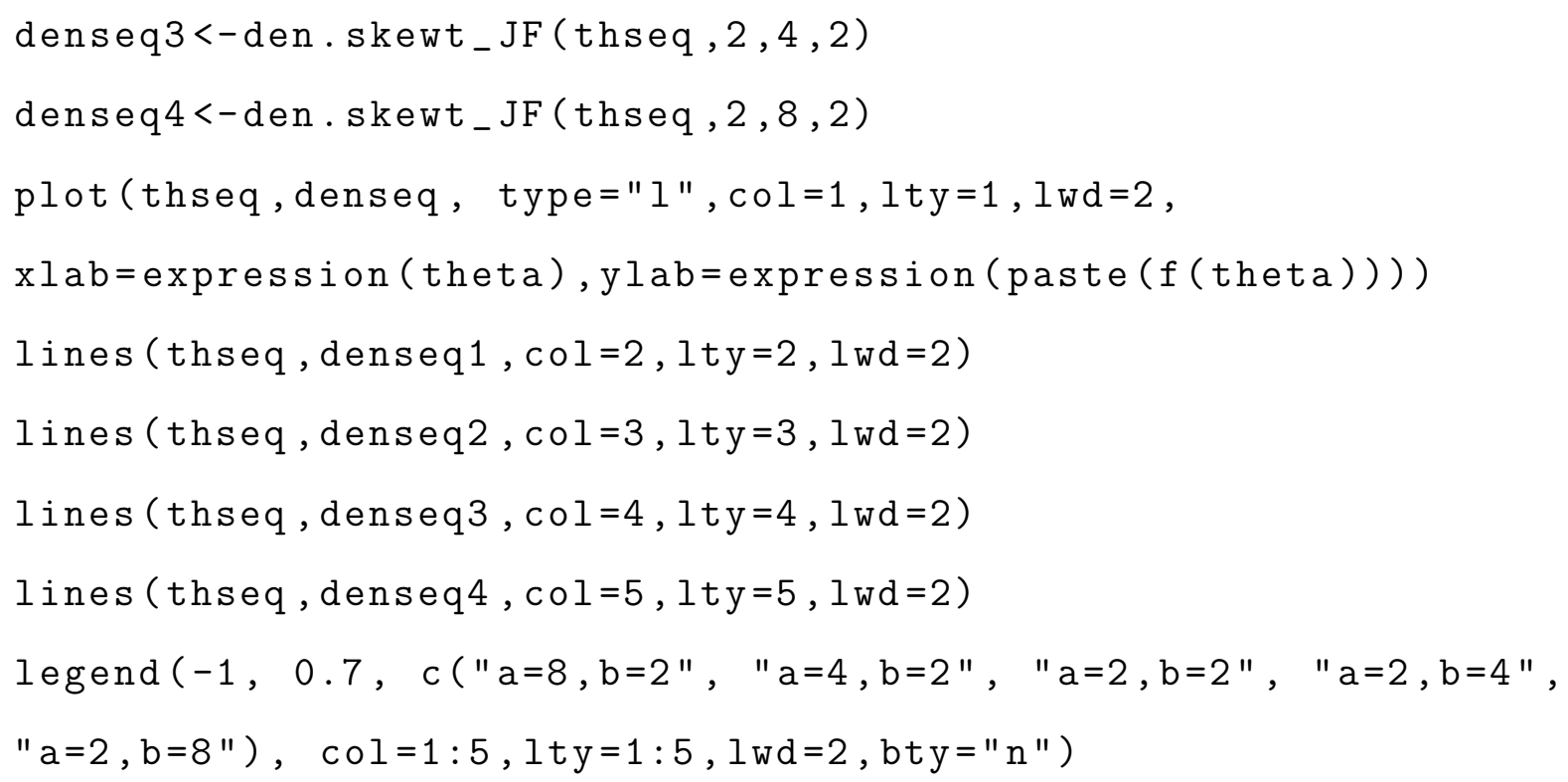

\#\# Reparametrized Density plot of Jones and Faddy skewed-t (2003) denR. skewt_JF<-function (theta, m, skp, v)

usk $<-\operatorname{skp} /(1+\operatorname{skp})$

$\mathrm{a}<-\mathrm{m} * \mathrm{usk} ; \mathrm{b}<-\mathrm{m}-\mathrm{a}$

den.skewt_JF(theta, a, b, v)

\}

thseq $<-\operatorname{seq}(-$ pi, pi, .1)

denseq<-denR.skewt_JF(thseq $, 10,4,2)$

denseq1<-denR. skewt_JF(thseq , 6, 2,2)

denseq $2<-$ denR. skewt_JF (thseq , 4, 1,2)

denseq $3<-$ denR. skewt_JF (thseq , $6, .5,2)$

denseq4<-denR.skewt_JF(thseq , $10, .25,2$ )

plot (thseq, denseq, type="l", $\operatorname{col}=1,1$ ty $=1,1 \mathrm{wd}=2$,

$\mathrm{xlab}=\operatorname{expression}($ thet $a), y l a b=\operatorname{expression}($ paste $(f($ theta $))))$

lines (thseq, denseq1, $\operatorname{col}=2,1$ ty $=2,1 \mathrm{wd}=2$ )

lines (thseq, denseq2, $\operatorname{col}=3,1$ ty $=3,1 \mathrm{wd}=2$ ) 
lines (thseq, denseq3, $\operatorname{col}=4,1$ ty $=4,1 \mathrm{wd}=2$ )

lines (thseq, denseq4, $\operatorname{col}=5,1$ ty $=5,1 \mathrm{wd}=2$ )

legend $(-1,0.7, c($ expression ( gamma=4), expression (gamma= $=2)$, expression ( gamma $==1)$, expression ( gamma $==.5)$, expression $($ gamma $==.25)), \operatorname{col}=1: 5,1$ ty $=1: 5,1 \mathrm{wd}=2$, bty="n" $)$

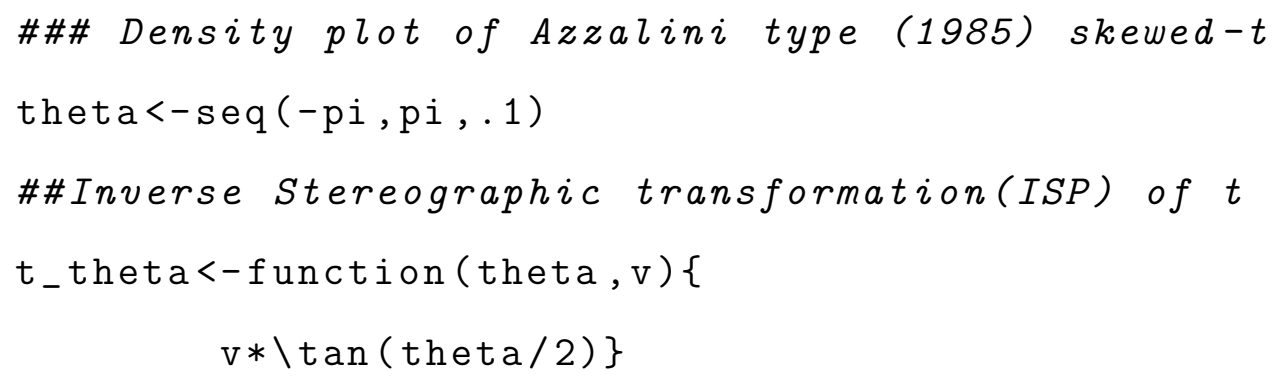


legend ( $-3,0.7, c($ expression (alpha=4), expression ( alpha==1), expression $(\mathrm{alpha}=0)$, expression $(\mathrm{alpha}=-4)$, expression $(\operatorname{alpha}==-1)), \operatorname{col}=1: 5,1$ ty $=1: 5,1 \mathrm{wd}=2, \mathrm{bty}=" \mathrm{n} ")$

\#\#\# Density plot of two-piece skewed-t

theta $<-$ seq $(-p i, p i, .1)$

\#\#Inverse Stereographic transformation(ISP) of $t$

t_theta<-function (theta, v) \{ $\mathrm{v} * \backslash \tan ($ theta/2)\}

\#\#density function of two-piece skewed-t with ISP den.skewt_TP<-function (theta, v, nu, gamma) \{

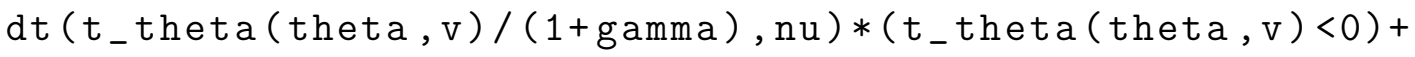
$\operatorname{dt}\left(t_{-}\right.$theta $($theta,$\left.v) /(1-\operatorname{gamma}), \mathrm{nu}\right) *\left(t_{-}\right.$theta $($theta,$\left.v)>=0\right)$ $\left.*(\mathrm{v} / 2) *(1+\backslash \tan (\operatorname{theta} / 2))^{-2}\right)$

\}

thseq<-seq $(-p i, p i, .1)$

denseq<-den.skewt_TP(theta , 2,10,.5)

denseq1<-den.skewt_TP(thseq $, 2,10, .3$ )

denseq $2<-$ den.skewt_TP(thseq $, 2,10,0)$

denseq $3<-$ den.skewt_TP (thseq , 2, 10, - .5)

denseq4<-den.skewt_TP (thseq , $2,10,-.3$ )

plot (thseq, denseq, type $=11$ ", $\operatorname{col}=1,1$ ty $=1,1 \mathrm{wd}=2$, $\mathrm{xlab}=\operatorname{expression}($ theta $), y l a b=\operatorname{expression}($ paste $(f($ theta $))))$

lines (thseq, denseq1, $\operatorname{col}=2,1$ ty $=2,1 \mathrm{wd}=2$ )

lines (thseq, denseq $2, \operatorname{col}=3,1$ ty $=3,1 \mathrm{wd}=2$ )

lines (thseq, denseq3, $\operatorname{col}=4,1$ ty $=4,1 \mathrm{wd}=2$ )

lines (thseq, denseq4, $\operatorname{col}=5,1$ ty $=5,1 \mathrm{wd}=2$ )

legend $(-3,0.4, c($ expression (gamma==.5), expression (gamma==.3), 
expression (gamma==0), expression $($ gamma $==-.5)$, expression $($ gamma $==-.3)), \operatorname{col}=1: 5,1$ ty $=1: 5,1$ wd $=2$, bty $=" n ")$

\section{A.3 R-code for maximum likelihood estimation}

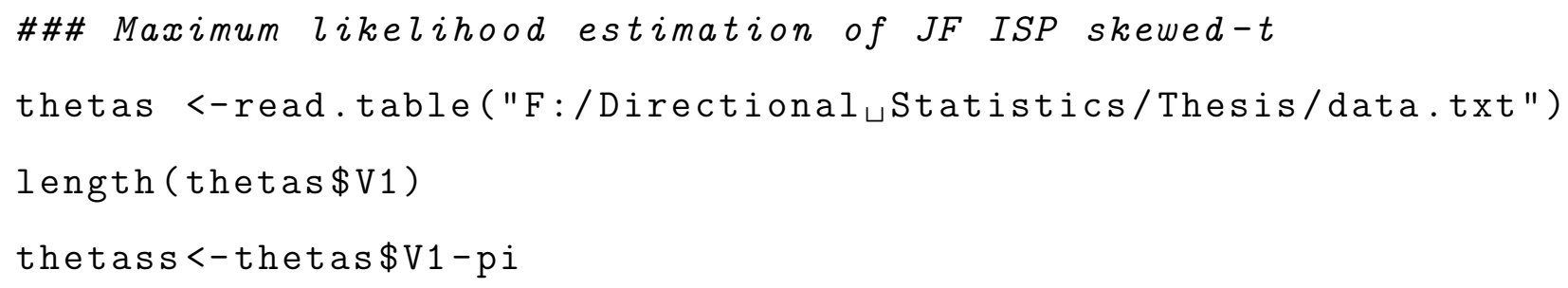




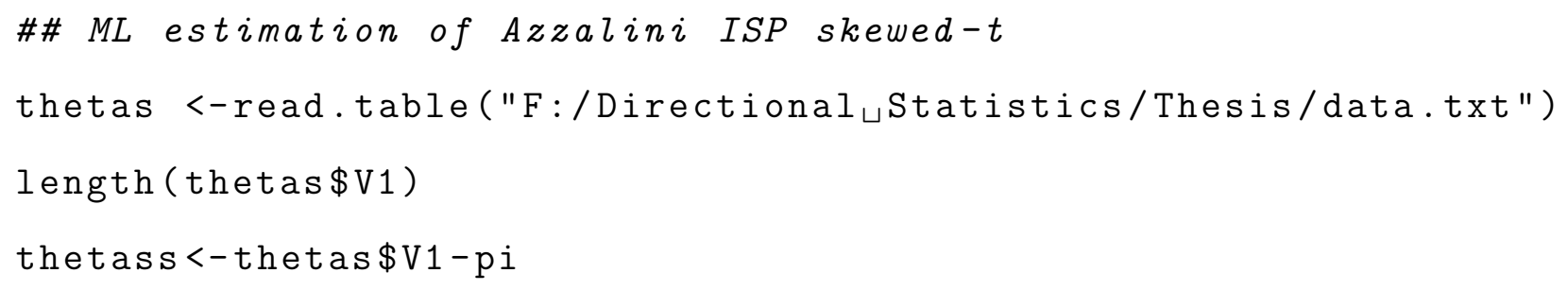

\#\#\# ML estimation of two-piece ISP skewed-t thetas <-read.table ("F:/DirectionalıStatistics/Thesis/data.txt") length (thetas \$V1) thetass $<-$ thetas $\$ V 1-$ pi 


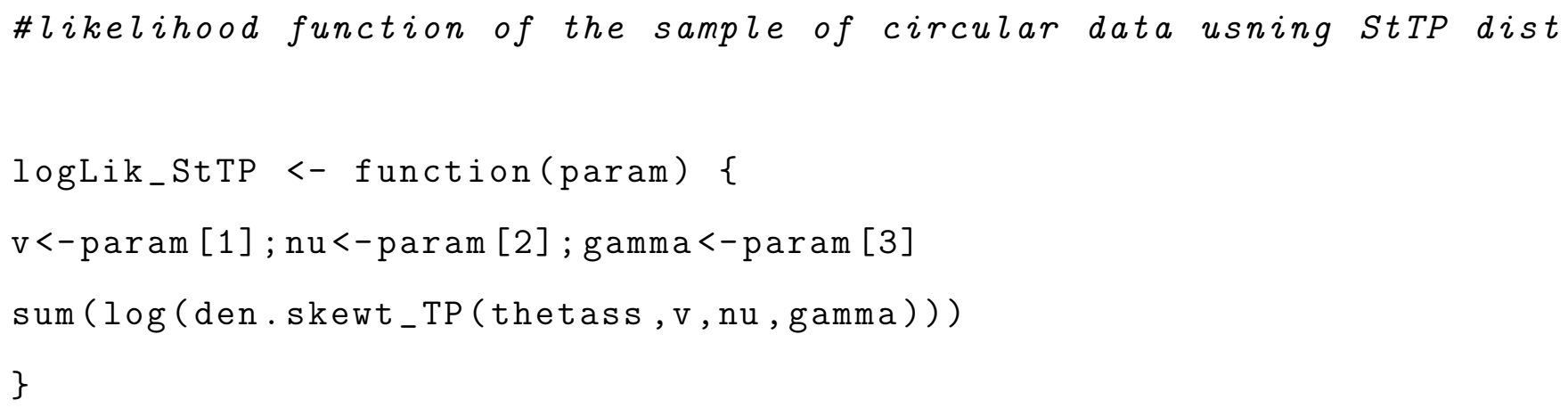

\section{A.4 R-code for histogram with fitted densities}

\#\# Histogram of bird migration heading data with fitted densities hist (thetass, prob=TRUE, breaks $=50, x l i m=c(-3,3), x l a b="$ Heading ( $r a d i a n$ $\operatorname{main}=$ NULL )

curve (denR.skewt_JF(x,5.08,2.11,2.62), col=1, lty=1, 1wd=2, add=T) curve (den.skewt_Azz ( $x, 1.88,2.98,1.58), \operatorname{col}=2,1 \mathrm{ty}=3,1 \mathrm{wd}=2$, add $=\mathrm{T}$ ) curve (den.skewt_TP ( $x, 2.08,3.36,-0.52), \operatorname{col}=4,1 \mathrm{ty}=2,1 \mathrm{wd}=2, \mathrm{add}=\mathrm{T})$ 
legend (-3, 0.55, c("St.JFபskewed-t", "St.Azz skewed-t",

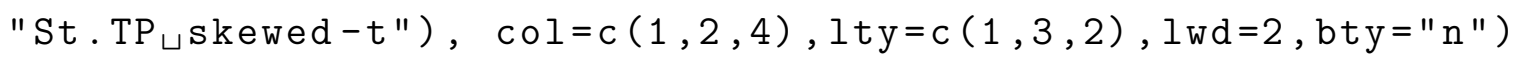

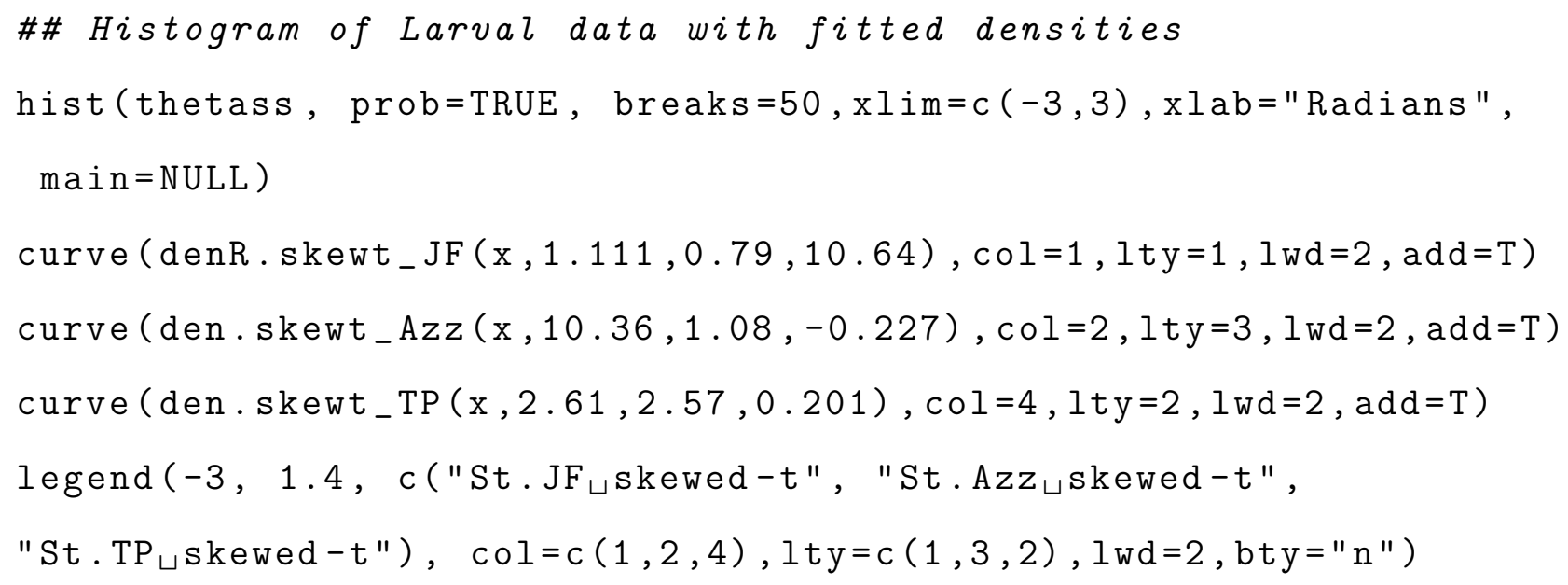

\#\# Histogram of Ants data with fitted densities

hist (thetass, prob=TRUE, breaks=40, $x l i m=c(-3,3)$, $x l a b=" R a d i a n s "$, main= NULL )

curve (denR.skewt_JF( $x, 0.87,1.074,4.94), \operatorname{col}=1,1$ ty=1, 1 wd $=2, \operatorname{add}=\mathrm{T})$ curve (den.skewt_Azz (x, 4.97,0.86,0.142), col=2, 1ty=3, 1wd=2, add=T) curve (den.skewt_TP ( $x, 1.91,1.324,0.000289), \operatorname{col}=4,1$ ty $=2,1 \mathrm{wd}=2, \operatorname{add}=\mathrm{T})$ legend (-3, 1.1, c("St.JFபskewed-t", "St.Azz skewed-t",

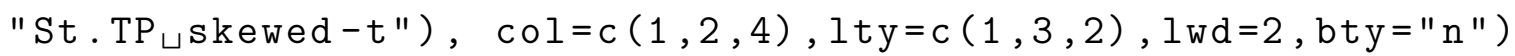

